\title{
Removal of the Pyrrolidine Substituent by Dehydrogenation of 4-Pyrrolidin-2-yl-3,4-dihydro- and 1,2,3,4-tetrahydroisoquinolines
}

\author{
Siavosh Mahboobi, Annette Karcher, Götz Grothus, Wolfgang Wagner, and Wolfgang Wiegrebe ${ }^{+* *}$ \\ Institute of Pharmacy, University, P. O. Box 1010 42, D-93040 Regensburg, Germany
}

Received October 1, 1993

Pyrrolidin-2-yl-groups located at C-4 of 3,4-dihydro- or 1,2,3,4-tetrahydroisoquinolines, respectively, are lost in the course of dehydrogenation of these isoquinoline derivatives. However, acyclically substituted isoquinolines, hydrogenated in ring B, 2-benzyl-4-(1-dimethylaminoethyl)-1,2,3,4tetrahydroisoquinoline, e.g., show loss of the amine group only by benzylic cleavage, affording 4-ethylisoquinoline. Scope and limitation of this reaction are determined using specifically substituted isoquinolines.
Abspaltung des Pyrrolidinylrestes bei der Dehydrierung von 4-Pyrrolidin-2-yl-3,4-dihydro- und 1,2,3,4-tetrahydroisochinolinen

C-4-Pyrrolidin-2-yl-3,4-dihydro- oder 1,2,3,4-tetrahydroisochinoline verlieren bei der Pd-katalysierten Dehydrierung diese Substituenten, während offenkettig substituierte ringhydrierte Isochinoline, z.B. 2-Benzyl-4-(1dimethylaminoethyl)-1,2,3,4-tetrahydroisochinolin nur die Aminfunktion unter Benzylspaltung zu 4-Ethylisochinolin abspalten. Die Grenzen dieser Reaktion werden anhand der Dehydrierung speziell substituierter Isochinolin-Derivate abgesteckt.
In the context of the removal of a $N$-methylpyrrolidine group by dehydrogenation from C-4 of the 3,4-dihydroisoquinoline skeleton in the course of the synthesis of the papaveraceae alkaloid macrostomine $(\mathbf{1})^{1)}$ we have reported on the dehydrogenation of simple 3,4-dihydro- and 1,2,3,4tetrahydroisoquinolines with pyrroline- and pyrrolidine increments at C-4: besides analogous losses we observed the expected dehydrogenation reaction (Scheme 3 in lit. ${ }^{2)}$ ) and rearrangements (Scheme 4 in lit. $\left.{ }^{2)}\right)^{2)}$.

Here we describe experiments performed in order to get some insight into scope and limitation of this abnormality.

\section{Results of Dehydrogenation Experiments}

These experiments were performed under standard conditions: $10 \% \mathrm{Pd} / \mathrm{C}$, tetraline, $190^{\circ}-210^{\circ} \mathrm{C}, \mathrm{N}_{2}, 1-2 \mathrm{~h}$. Whilst the 1-methyl-4-(2,5-dihydro- $N$-methylpyrrol-2-yl)3,4-dihydroisoquinoline $\mathbf{2}$ is nicely aromatized in the pyrroline- as well as in the dihydroisoquinoline-moiety (Scheme 5 in Lit. $^{2)}$ ), the 3,4-dihydro-5 $\mathrm{H}$-pyrrole group and the 3,4,5,6-tetrahydropyridine increment at C-4 of the (aromatic) isoquinolines 3 and $\mathbf{4}$, respectively, remain unchanged.

This holds true also for the pertinent sec amines 5 and $\mathbf{6}$, and also the corresponding $N$-methylated pyrrolidine- and piperidine-derivatives 7 and 8 are not dehydrogenated. In all theses cases the heterocyclic ring at C-4 of the isoquinoline systems is not split off. As already observed by See$b a c h^{3)}$ in his synthesis of macrostomine (1), the loss of the pyrrolidine increment is prevented by $\mathrm{N}$-formylation: cpds. 9 and 10 are dehydrogenated in the isoquinoline ring only, affording 9a and 10a. - From these experiments we can conclude that

+) Dedicated to Prof. Dr.h.c. A. Brossi, NIH, USA, on the occasion of his 70th birthday. a) the non-bonding electron pair at the $\mathrm{N}$-atom of the attached ring is a prerequisite for this cleavage. Here only the tetrahydroisoquinoline system is dehydrogenated.

b) the loss of the fully hydrogenated $N$-heterocycle is synchronous with the dehydrogenation of the 3,4-dihydro- or 1,2,3,4-tetrahydroisoquinoline nucleus.

In order to check point $b$ ) we have prepared cpd. 11 with two substituents at C-4. Here, the 3,4-dihydroisoquinoline system as well as the $N$-methylpyrrolidine group should survive the dehydrogenation conditions, because we expected aromatization not to occur. This assumption, however, turned out to be wrong: there was loss of the pyrrolidine increment in $\mathbf{1 1}$ (for the fate of this group cf. lit. ${ }^{2)}$ ), and the 3,4-dihydroisoquinoline system was aromatized affording the 1,4-dimethylisoquinoline 12. Curiously enough this reaction took place even without $\mathrm{Pd}$ as a catalyst by thermal cleavage only. So it seemed reasonable to assume that gain of aromatization energy is the driving force for this cleavage, but this assumption is disputable because dehydrogenation of 6,7-dimethoxy-1-methyl-4-( $N$-methylpyrrolidin-2yl)-3,4-dihydroisoquinoline (13) under standard conditions affords inter alia 6,7-dimethoxy-1-methyl-3,4-dihydroisoquinoline $(\mathbf{1 4})^{2}$. Our speculation that subsequent hydrogenation of the isoquinoline system to the pertinent 3,4-dihydro-derivative due to dehydrogenation of tetraline ${ }^{4)}$ had occured, was discarded because a pertinent experiment with isoquinoline itself led to dimerization only (not shown).

A $N$-methylpyrrole ring at C-4 does not interfere with dehydrogenation of the 1,2,3,4-tetrahydro- or the 3,4-dihydroisoquinoline system (cf. 15, 16).

Up to now we have described dehydrogenation of molecules having the amine function as part of a ring system bound to $\mathrm{C}-4$ of the isoquinoline by its $\mathrm{C}-2$. Therefore, we dehydrogenated analogous open chain compounds. 
<smiles></smiles>

(+/-)-Macrostomine 1<smiles>[R]N1CCCC1c1cncc2ccccc12</smiles>

5: $n=1 ; R=H$ 6: $n=2 ; R=H$

7: $n=1 ; R=\mathrm{CH}_{3}$<smiles>COc1cc2c(cc1OC)C(C1C=CCN1C)CN=C2C</smiles>

2

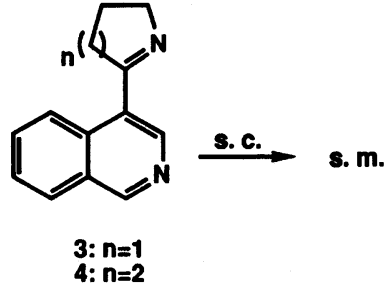<smiles>O=CN1CCCC1c1cncc2ccccc12</smiles>

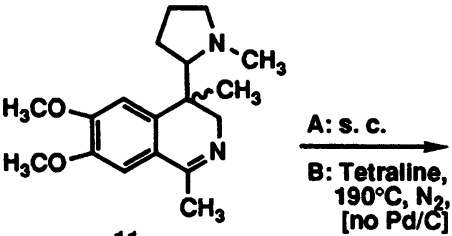

[no Pd/C]<smiles>COc1cc2c(C)cnc(C)c2cc1OC</smiles>

12<smiles>COc1cc2c(cc1OC)C(C1CCCN1C)CN=C2C</smiles>

13<smiles>COc1cc2c(cc1OC)C(C)=NCC2</smiles>

14<smiles>COc1cc2c(cc1OC)C(c1cccn1C)CNC2Cc1ccc2c(c1)OCO2</smiles>

15<smiles>COc1cc2c(-c3cccn3C)cnc(Cc3ccc4c(c3)OCO4)c2cc1OC</smiles>

$16 a$<smiles>COc1cc2c(cc1OC)C(c1cccn1C)CN=C2Cc1ccc2c(c1)OCO2</smiles>

16

\section{Scheme 1}

4-(1-Dimethylaminoethyl)isoquinoline (17) shows benzylamine cleavage (tetraline as a hydrogen source) affording 4-ethylisoquinoline $(18)^{5}$. Cpd. 18 also arose from the $N$ benzylated tetrahydroisoquinoline 19. Because Seebach et $a l .{ }^{3)}$ have found that $N$-benzylated tetrahydroisoquinolines are debenzylated hydrogenolytically during dehydrogenation, there are no objections against the reaction of 19.

If we omit one component of the standard conditions (Scheme 2, cpd. 17, experiments B-E), no benzylamine cleavage occurs, indicating that this cleavage is a hydrogenolytic one. - Contrary to the 4-pyrrolidinyl-substituted 1,4- dimethyl-3,4-dihydroisoquinoline 11 the open-chain substituted tetrahydroisoquinoline $\mathbf{2 0}$ (mixture of diastereomers) is dehydrogenated to the 3,4-dihydroisoquinoline 21 which is stable under standard conditions. Here no thermal cleavage occured (cf. cpd. 11).

We hypothesize from these experiments that the title reaction is restricted to cyclic amines with $\mathrm{N}$ in the 2-position, substituting C-4 of the 3,4-dihydro- or 1,2,3,4-tetrahydroisoquinoline. Probably - but consider the thermal degradation in the case of cpd. 11 - the hydrogenolytic cleavage depends on a more or less flat orientation of the said com- 

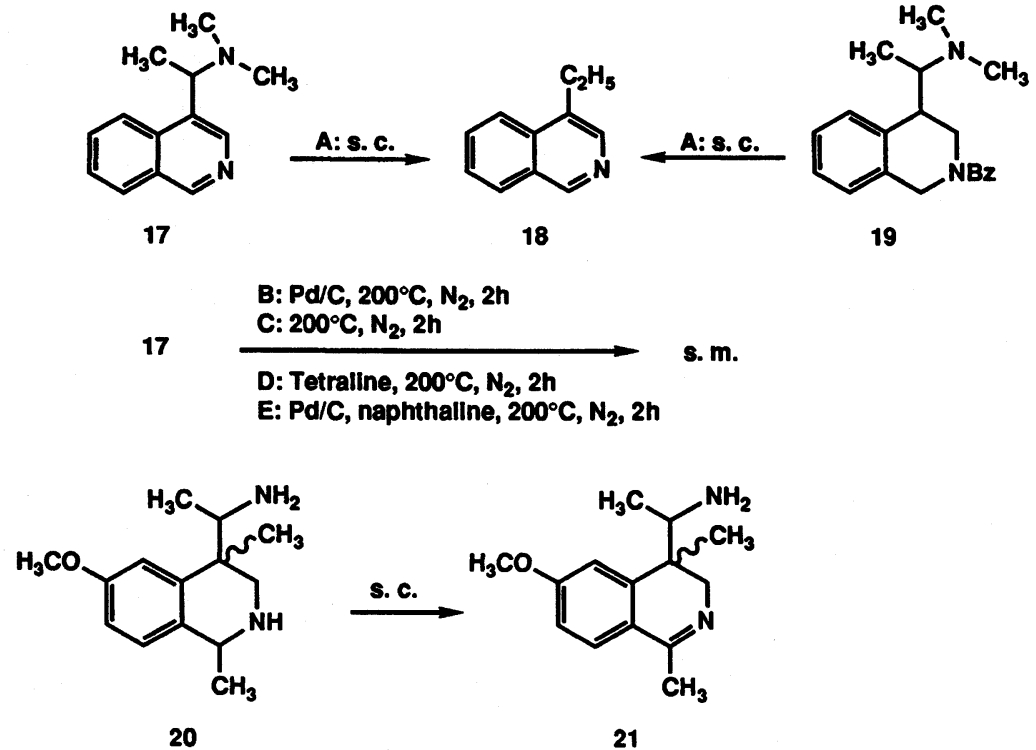

20

21
s. c. : standard conditions
$\mathrm{Pd} / \mathrm{C}$, tetraline, $190-210^{\circ} \mathrm{C}, \mathrm{N}_{2}, 1-2 \mathrm{~h}$
s. m. : starting material

Scheme 2

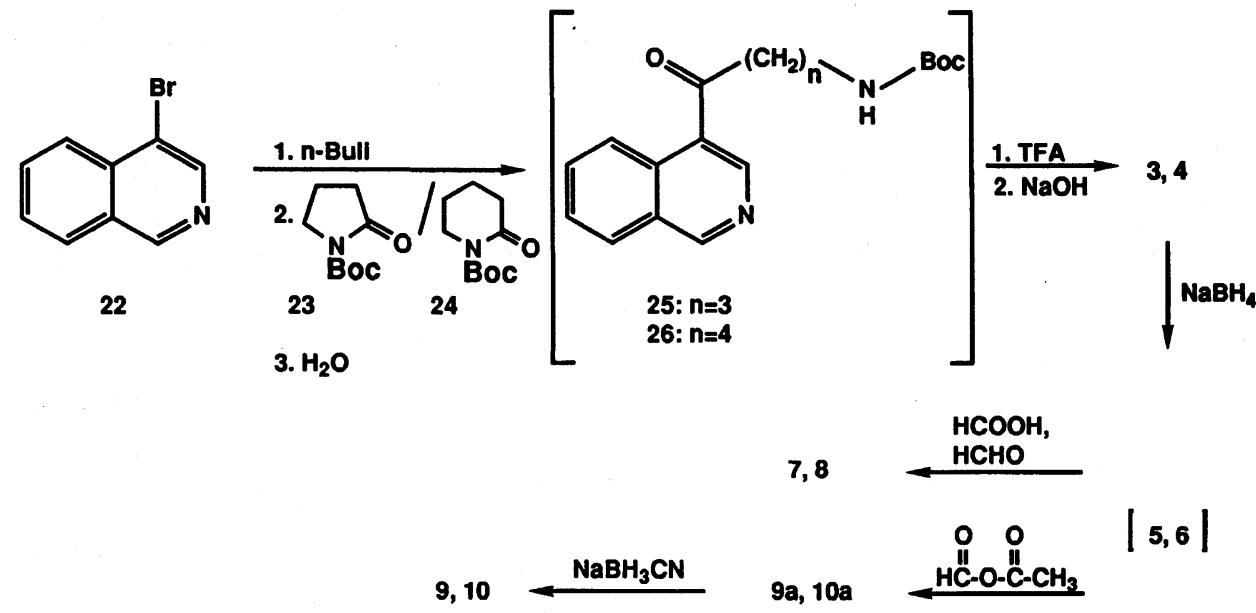

Scheme 3

pounds at the surface of the catalyst, facilitated by the somewhat restricted conformational mobility of the amine being part of a ring, and is triggered by the gain of resonance energy during aromatization of the hydrogenated ring $B$ of the isoquinoline system. Up to now we have no chance to check this speculation.

\section{Synthesis of test compounds}

a) Ring substituents

The isoquinoline derivatives substituted at C-4 by a $N$ formyl- or $\mathrm{N}-\mathrm{CH}_{3}$-pyridine- or piperidine-ring were prepared according to Scheme 3:
4-Bromoisoquinoline $(\mathbf{2 2})^{5)}$ was deprotonated to its anion which reacted with $N$-Boc-pyrrolidin-2-one $\left.(23)^{6}\right)$ or $N$ Boc-piperidin-2-one $(\mathbf{2 4})^{7)}$ affording the urethanes 25 and 26, respectively. Deprotection and subsequent intramolecular condensation led to the imines 3 and 4 which were reduced to the pertinent sec amines 5 and 6. EschweilerClarke methylation yielded the $N$-methylpyrrolidine- $/ N$ methylpiperidine-substituted isoquinolines 7 and 8 , whilst $N$-formylation to 9a, 10a with subsequent reduction afforded the $N$-formylated tetrahydroisoquinolines 9 and $\mathbf{1 0}$.

For the synthesis of the twofold substituted 3,4-dihydroisoquinoline 11 we first followed Seebach's concept of alkylation of twofold deprotonated 2-phenyl-1-nitroethanes $^{8)}$, but in addition to $\mathrm{C}-2, \mathrm{C}-1$ of the nitroethane 27 
<smiles>COc1ccc(C(C[N+](=O)[O-])c2cccn2C)cc1OC</smiles>

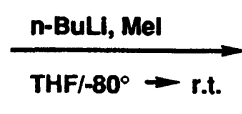<smiles>COc1ccc(C(C)(C[N+](=O)[O-])c2cccn2C)cc1OC</smiles><smiles>COc1ccc(C(c2cccn2C)C(C)[N+](=O)[O-])cc1OC</smiles>

29

+ starting material $\quad 36 \%$<smiles>COc1ccc(C(C#N)c2cccn2C)cc1OC</smiles>

30<smiles>COc1ccc(C(C)(C)C(C)(C)c2cccn2C)cc1OC</smiles>

31

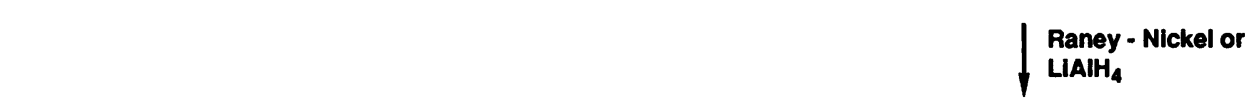

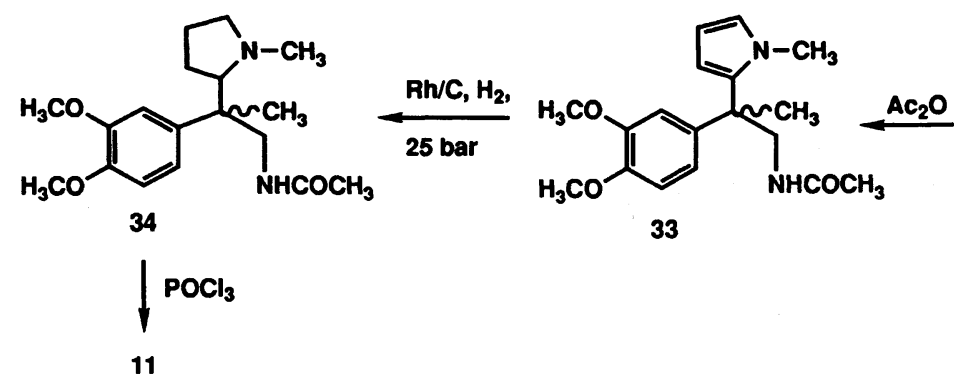<smiles>COc1ccc(C(C)(CN)c2cccn2C)cc1OC</smiles>

32

Scheme 4

was methylated, probably due to the two substituents at the benzylic position (Scheme 4). Besides starting material 27 we obtained the methyl derivatives 28 and 29. So we went on with the nitril $\mathbf{3 0}$ which was nicely methylated in $\alpha$-position, yielding nitril 31. Further steps (Scheme 4) are routine.

\section{b) Open chain substituents}

Reaction of the isoquinoline-C-4-anion, obtained from 4bromoisoquinoline (22) (vide supra) with acetaldehyde produced the sec carbinol $\mathbf{3 5}^{9)}$ which was dehydrogenated to the ketone $\mathbf{3 6}^{9}$. . Reductive amination with dimethylamine afforded the tert. amine 17 (Scheme 5).

Ketone 36 when treated analogously with methylamine yielded the sec. amine 37 which was $N$-formylated (38) and reduced in the isoquinoline part.

Here a mixture of $\mathbf{3 9}$ and $\mathbf{4 1}$ (main isomer) was obtained, due to a shift of the $N$-formyl group (Scheme 6). This was indicated by the MS-fragments $\mathrm{CH}_{3}-\mathrm{CH}=\mathrm{NCH}_{3}(\mathrm{CHO})(\mathrm{m} / \mathrm{z}$ $=86$ of 39) and $\mathrm{CH}_{3}-\mathrm{CH}=\mathrm{NH}\left(\mathrm{CH}_{3}\right)(\mathrm{m} / \mathrm{z}=58$ of 41) with high rel. intensities, whilst $\mathrm{M}^{+\cdot}$ at $\mathrm{m} / \mathrm{z}=218$ indicated that reduction of the isoquinoline increment had occured. Loss of $58 \mathrm{mu}\left(\mathrm{C}_{2} \mathrm{H}_{4} \mathrm{NO}\right)$ from 39 is unlikely and was excluded by HR-MS $\left(\mathrm{C}_{3} \mathrm{H}_{8} \mathrm{~N}\right.$ calcd. 58.06567; $\mathrm{C}_{2} \mathrm{H}_{4} \mathrm{NO}$ calcd. 58.02929; found 58.06571). Reduction of this mixture with $\mathrm{LiAlH}_{4}$ led to a mixture of diastereomers with $\mathrm{M}^{+\cdot}$ at $\mathrm{m} / \mathrm{z}=$ 202 instead of $\mathrm{m} / \mathrm{z}=204$. Probably the benzo[f]-1,3-diazabicyclo[3.3.1]nonan $\mathbf{4 2}$ had been formed. This requires loss of water from the $\alpha$-hydroxyamino group of $\mathbf{4 0}$ yielding an iminium ion and subsequent reduction.

As we could not avoid this formyl-migration, we have benzylated cpd. 38 at the isoquinoline- $N(\mathbf{4 3})$ and reduced the isoquinolinium ion to the 1,2,3,4-tetrahydro-derivative 44. No problems arose with this compound when we reduced the $N$-formyl increment to the tert amine side chain, but 
22

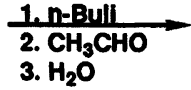<smiles>CC(O)c1cncc2ccccc12</smiles>

35
$\mathrm{MnO}_{2}$<smiles>C=Cc1ccccc1C(=C)C(=C)C</smiles>

$\mathrm{HN}\left(\mathrm{CH}_{3}\right)_{2}$

$\mathrm{NaBH}_{3} \mathrm{CN}$

17

Scheme 5<smiles>CC(c1cncc2ccc(C(C)(C)N)cc12)N(C)C</smiles>

37

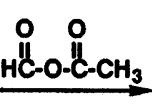

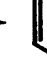

36

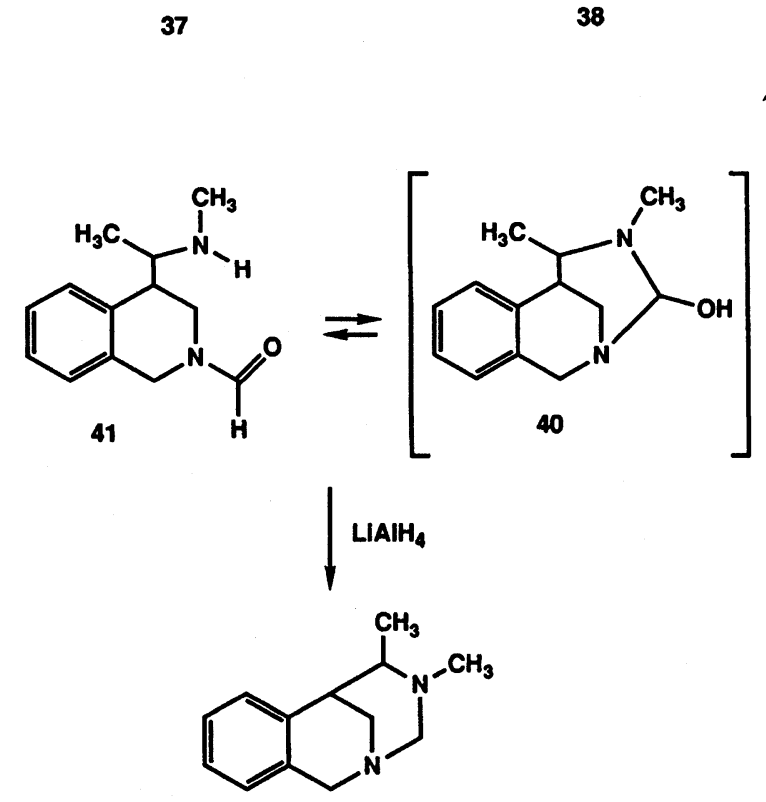

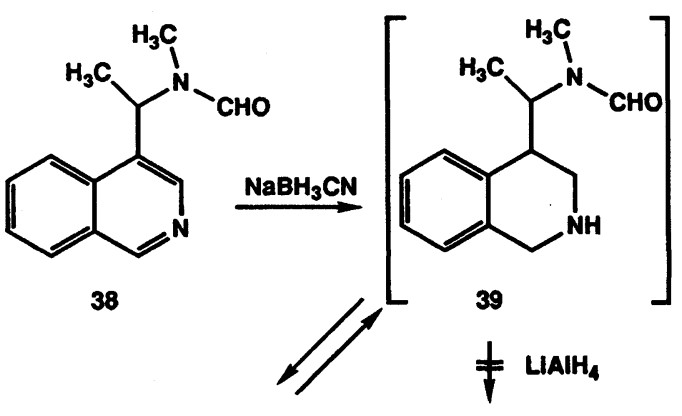<smiles>CC(C1CNCc2ccccc21)N(C)C</smiles>

42 mixture of diastereomeres

Scheme 6

all our efforts to cleave the $\mathrm{N}$-benzyl moiety hydrogenolytically failed (Hartung ${ }^{10)}$, Grewe ${ }^{11)}$, Cava12), Yagi ${ }^{13)}$,

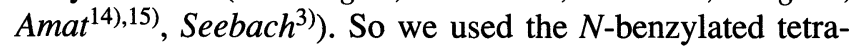
hydroisoquinoline 19 directly for the dehydrogenation experiment (vide supra).

4-(1-Aminoethyl)-6-methoxy-1,4-dimethyl-1,2,3,4tetrahydroisoquinoline (20) was synthesized starting from ketone $\mathbf{4 5}^{16}$ ) (Scheme 8) which was converted into the 1,3dithiolane 46 and the 1,3-dithiane 47, respectively. Methylation occured in $\alpha$-position of the nitril, but in addition a $\mathrm{SCH}_{3}$-group arose, resulting from base-catalyzed $\beta$-elimination after $S$-methylation $(\mathbf{4 8}, \mathbf{4 9})$. This ring cleavage was reversed for 48 by treating it with 1,2-dimercaptoethane $/ \mathrm{BF}_{3}$, yielding $\mathbf{5 0}$. Reduction of the nitril $\mathbf{5 0}$ afforded the prim amine 51 which was acetylated (52). BischlerNapieralski reaction at room temp. produced the 3,4-dihy- droisoquinoline 53 which was reduced as usual (54). The dithiolane ring was removed by $\mathrm{Hg}\left(\mathrm{ClO}_{4}\right)_{2}{ }^{17)}$. The resulting ketone 55 could not be transformed to a $N$-dimethylaminoethyl increment under various conditions (cf. preparation of $\mathbf{1 7}$ for comparison). So we prepared the prim amine via the $O$-methyloxime 56 which was reduced by diborane.

In order to avoid ring cleavage of the dithiolan/dithian system in 46 and 47, respectively, these molecules were treated with $\mathrm{H}_{3} \mathrm{CI}$ under the conditions shown in Scheme 9, but these efforts failed: large scale experiments yielded the $\alpha, \beta$-unsaturated nitril 57. Further methylation afforded the $C$ - $\alpha$-methylated nitrils 48 and 49 which were directly obtained when using smaller quantities of $\mathbf{4 6}$ or $\mathbf{4 7}$. An equimolar concentration of $\mathrm{H}_{3} \mathrm{CI}$ at room temp. led to the thioketone 58 by an unknown mechanism ${ }^{18)}$. 


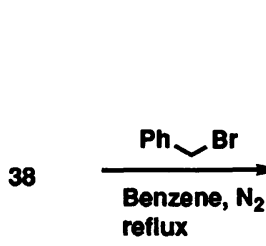

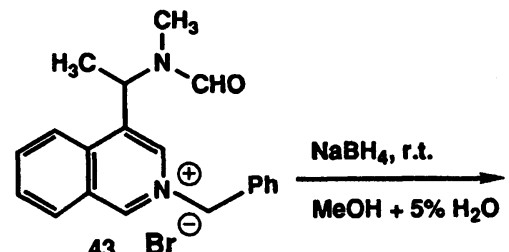

$43 \mathrm{Br}^{\ominus}$<smiles>CC(C1CN(Cc2ccccc2)Cc2ccccc21)N(C)C=O</smiles>

44

LIAIH ${ }_{4}, N_{2}$ THF, r.t.

Scheme 7<smiles>COc1cccc(C(C#N)C(C)=O)c1</smiles><smiles></smiles>

45<smiles>COc1cccc(C(C)(C)C2(C)C=CS2)c1</smiles><smiles>COc1cccc(C(C)(CN)C23CCC(C2)S3)c1</smiles>

51<smiles>CCC1(C)c2cc(OC)ccc2SC=CC=C2C(C)NCC21C</smiles><smiles>CC[18OH]</smiles>

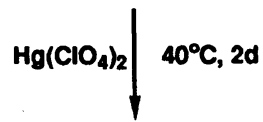<smiles>COc1ccc2c(c1)C(C)(C(C)=O)CNC2C</smiles><smiles>COc1cccc(C(C#N)C(C)(S)SC2CCCC2)c1</smiles><smiles>CCOC([Mg])=[W]</smiles><smiles>C=C(SCCSC)C(C)(C)c1cccc(OC)c1</smiles><smiles>COc1cccc(C(C)(CNC(C)=O)C2(C)C=CS2)c1</smiles>

52

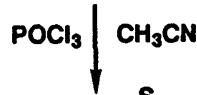<smiles></smiles><smiles>CON=C(C)C1(C)CNC(C)c2ccc(OC)cc21</smiles>

20

Scheme 8 


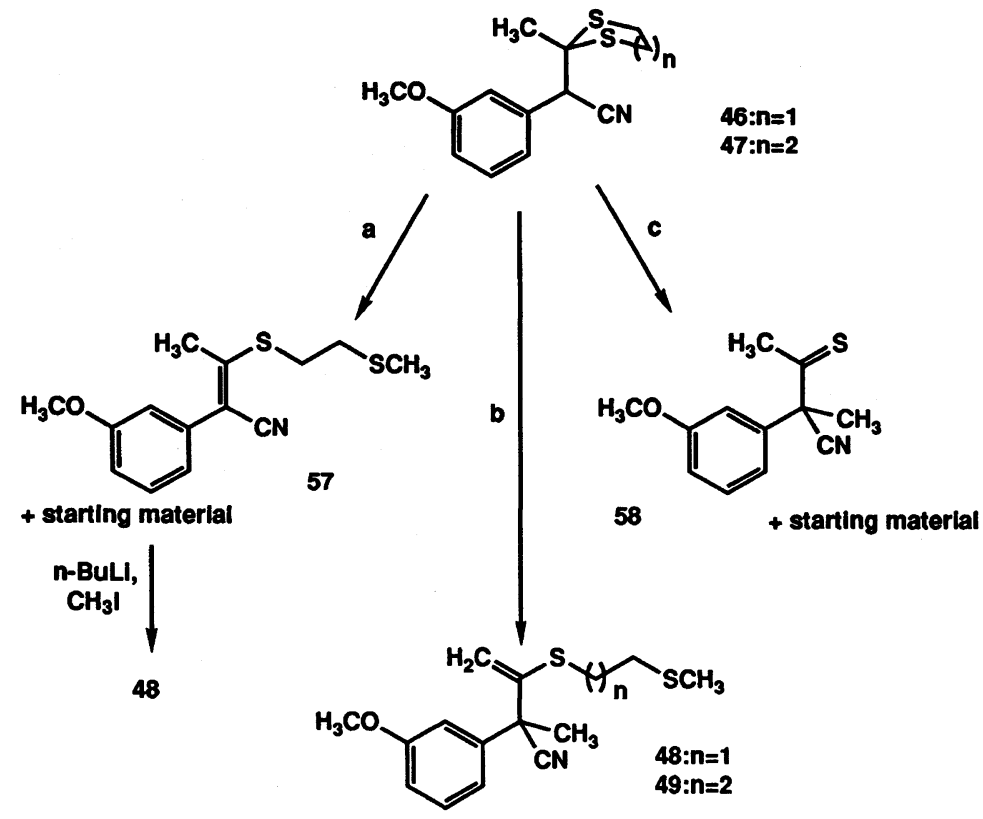

\begin{tabular}{l|l|l|l|l|l} 
run & $\mathrm{CH}_{3} \mathrm{l}$ & $46 / 47$ & solvent & time & temp. \\
\hline a & 2.1 mole-eq. & $50 \mathrm{~g}$ & $\begin{array}{l}\text { THF/Et } 2 \mathrm{OI} \\
\text { HMPT }\end{array}$ & $10 \mathrm{~h}$ & $-30^{\circ} \mathrm{C}$ \\
\hline $\mathrm{b}$ & 2.1 mole-eq. & $<10 \mathrm{~g}$ & $\begin{array}{l}\text { THF/Et } \\
\text { TMEO }\end{array}$ & $2 \mathrm{~h}$ & $-30^{\circ} \mathrm{C}$ \\
\hline $\mathrm{C}$ & 1 mole-eq. & $<10 \mathrm{~g}$ & $\begin{array}{l}\text { THF/Et }_{2} \mathrm{O} \\
\text { TH }\end{array}$ & $12 \mathrm{~h}$ & $+25^{\circ} \mathrm{C}$
\end{tabular}

Scheme 9

\section{Experimental Part}

General remarks: lit. ${ }^{2)}$.- UV-spectra: $\mathrm{MeOH}$, if not stated otherwise.- ${ }^{1} \mathrm{H}-$ NMR-spectra: $90 \mathrm{MHz}$, in $\mathrm{CDCl}_{3}$, if not stated otherwise.- Temp. in ${ }^{\circ} \mathrm{C}$.Drying over $\mathrm{Na}_{2} \mathrm{SO}_{4}$ - Column chromatography (cc): $\mathrm{SiO}_{2}$.

\section{$\boldsymbol{A}$. Preparation of the compounds}

4-(Pyrrolin-2-yliden)-isoquinoline (3)

To $17 \mathrm{ml}$ (27.2 mmole) of n-BuLi (15\% in hexane) in $50 \mathrm{ml}$ of dry $\mathrm{Et}_{2} \mathrm{O} / \mathrm{THF}$ (1:1) were added under $\mathrm{N}_{2} 5.6 \mathrm{~g}$ (27.0 mmole) of 4-bromoisoquinoline (22) in $30 \mathrm{ml}$ of dry THF and $5 \mathrm{~g}(27.0 \mathrm{mmole})$ of $N$-(tertbutoxycarbonyl)-pyrrolidin-2-one (23) in $10 \mathrm{ml}$ of dry THF drop by drop at $-90^{\circ}$ with stirring. Stirring was continued for $1 \mathrm{~h}$ at $-90^{\circ}$ and for $2 \mathrm{~h}$ at $-75^{\circ}$. At this temp. the mixture was quenched by dropwise addition of 20 $\mathrm{ml}$ of $2 \mathrm{~N} \mathrm{HCl}$. After removal of the cooling bath the mixture was allowed to warm to room temp. and extracted with $\mathrm{Et}_{2} \mathrm{O}$. The extracts were washed with $10 \%$ aqueous $\mathrm{NaHCO}_{3}$, dried, and evaporated to give a brownish oil (94\%) of 25 (not purified). After direct treatment with $10 \mathrm{ml}$ of trifluoroacetic acid at $0^{\circ}$ and stirring for $2 \mathrm{~h}$ at room temp. $50 \mathrm{ml}$ of water were added and the mixture was made alkaline by careful addition of $5 \%$ $\mathrm{NaOH}$. Work-up gave a brownish oil. Chromatography (cyclohexane:ethyl acetate $=1: 1)$ and recrystallization from petrolether gave $210 \mathrm{mg}(4 \%)$ analytically pure 3 , colourless needles, mp. $68^{\circ}$.- $\mathrm{C}_{13} \mathrm{H}_{12} \mathrm{~N}_{2}$ (196.2) Calcd. C 79.6 H 6.16 N 14.3 Found C 79.3 H 6.14 N 14.1.- UV: $\lambda \max (\log \varepsilon)=$ 221 (4.35); 284 (3.45); $321 \mathrm{~nm}$ (3.45).- IR (KBr): $\tilde{v}=2970 \mathrm{~cm}^{-1}(\mathrm{CH})$. ${ }^{1} \mathrm{H}-\mathrm{NMR}: \delta(\mathrm{ppm})=2.05-2.15\left(\mathrm{~m} ; 2 \mathrm{H}, \mathrm{CH}_{2}\right), 3.10-3.20\left(\mathrm{~m} ; 2 \mathrm{H}, \mathrm{CH}_{2}\right)$, 4.20-4.30 (m; $2 \mathrm{H}, \mathrm{CH}_{2}$ ), 7.60-8.00 (m; $3 \mathrm{H}$ aromat.), 8.75 (s; $1 \mathrm{H}$ aromat.), 9.20-9.25 (m; $2 \mathrm{H}$ aromat.).- $\mathrm{MS}: \mathrm{m} / \mathrm{z}=196\left(98 \%, \mathrm{M}^{+\bullet}\right), 195(100$, $\mathrm{M}-\mathrm{H})^{+}$.

\section{4-(Piperidein-2-yliden)-isoquinoline (4)}

Following the procedure for 3, $5.4 \mathrm{~g}(27 \mathrm{mmole}) \mathrm{N}$-(tert-butoxycarbonyl)-piperidin-2-one (24) gave $350 \mathrm{mg}(6 \%) 4$ as a light amber oil.$\mathrm{C}_{14} \mathrm{H}_{14} \mathrm{~N}_{2}$ (210.3).- UV: $\lambda \max (\log \varepsilon)=217(4.43) ; 273$ (3.48); $322 \mathrm{~nm}$ (3.48).- IR (film): $\tilde{\mathrm{v}}=2940 \mathrm{~cm}^{-1}(\mathrm{CH}) .{ }^{1} \mathrm{H}-\mathrm{NMR}: \delta(\mathrm{ppm})=1.60-2.00(\mathrm{~m}$; $\left.4 \mathrm{H}, \mathrm{CH}_{2}\right), 2.50-2.65\left(\mathrm{~m} ; 2 \mathrm{H}, \mathrm{CH}_{2}\right), 3.85-4.10\left(\mathrm{~m} ; 2 \mathrm{H}, \mathrm{CH}_{2}\right), 7.50-8.35(\mathrm{~m}$; $4 \mathrm{H}$ aromat.), 8.60 (s; $1 \mathrm{H}$ aromat.), 9.25 (s; $1 \mathrm{H}$ aromat.). $\mathrm{MS}: \mathrm{m} / \mathrm{z}=210$ $\left(68 \%, \mathrm{M}^{+}\right), 209(100, \mathrm{M}-\mathrm{H})^{+}$.

\section{4-(N-Methyl-pyrrolidin-2-yl)-isoquinoline (7)}

To a solution of $100 \mathrm{mg}(0.51 \mathrm{mmole})$ of 3 in $2 \mathrm{ml}$ of dry $\mathrm{MeOH}$ were added $38 \mathrm{mg}(1.0 \mathrm{mmole})$ of $\mathrm{NaBH}_{4}$ under $\mathrm{N}_{2}$ at $0^{\circ}$ in portions. After stirring for $3 \mathrm{~h}$ at $0^{\circ} 5 \mathrm{ml}$ of water were added and the mixture was made alkaline and extracted with $\mathrm{Et}_{2} \mathrm{O}$. The extracts were washed with brine, dried, and evaporated to give a brownish oil (5). Without purification raw 5 was refluxed in $5 \mathrm{ml}$ of $35 \%$ aqueous formaldehyde and $3 \mathrm{ml}$ of formic acid for $20 \mathrm{~h}$. After addition of $2 \mathrm{ml}$ of $2 \mathrm{~N} \mathrm{HCl}$ the mixture was concentrated in vacuo, made alkaline by $5 \% \mathrm{NaOH}$ and extracted with $\mathrm{Et}_{2} \mathrm{O}$. The extracts were washed with brine, dried, and evaporated: brownish oil (94\%). Chromatography (MeOH:ethyl acetate $=1: 1$ ) gave $53 \mathrm{mg}(49 \%) 7$ as an amber oil.- $\mathrm{C}_{14} \mathrm{H}_{16} \mathrm{~N}_{2}$ (212.3).- UV: $\lambda \max (\log \varepsilon)=218$ (4.58); 272 (3.88); 310 (3.78); $323 \mathrm{~nm}$ (3.88).- IR (film): $\widetilde{v}=2960 \mathrm{~cm}^{-1}(\mathrm{CH}) .{ }^{1} \mathrm{H}-\mathrm{NMR}(250$ $\mathrm{MHz}): \delta(\mathrm{ppm})=1.84-2.09(\mathrm{~m} ; 3 \mathrm{H}, \mathrm{CH}), 2.27\left(\mathrm{~s} ; 3 \mathrm{H}, \mathrm{N}-\mathrm{CH}_{3}\right), 2.32-2.46$ (m; $2 \mathrm{H}, \mathrm{CH}), 3.31-3.37(\mathrm{~m} ; 1 \mathrm{H}, \mathrm{CH}), 3.67-3.73(\mathrm{~m} ; 1 \mathrm{H}, \mathrm{CH}), 7.56-7.73$ (m; $2 \mathrm{H}$ aromat.), 8.06 (d; J = 8.4 Hz, $1 \mathrm{H}$ aromat.), 8.33 (d; J = $8.4 \mathrm{~Hz}, 1 \mathrm{H}$ aromat.), 8.64 (s; $1 \mathrm{H}$ aromat.), 9.16 (s; $1 \mathrm{H}$ aromat.).- MS: $\mathrm{m} / \mathrm{z}=212$ (17\%, $\left.\mathrm{M}^{+^{\bullet}}\right), 84\left(100, \mathrm{C}_{5} \mathrm{H}_{10} \mathrm{~N}\right)^{+}$. 
4-(N-Methyl-piperidin-2-yl)-isoquinoline (8)

Following the procedure for $7,100 \mathrm{mg}(0.48 \mathrm{mmole})$ of 4 gave a brownish oil (6) which afforded $56 \mathrm{mg}(52 \%) 8$ as a light amber oil.- $\mathrm{C}_{15} \mathrm{H}_{18} \mathrm{~N}_{2}$ (226.3).- UV: $\lambda \max (\log \varepsilon)=220$ (4.60); 272 (3.71); 310 (3.61); $323 \mathrm{~nm}$ (3.72).- IR (film): $\widetilde{v}=2940 \mathrm{~cm}^{-1}(\mathrm{CH}) .{ }^{1} \mathrm{H}-\mathrm{NMR}(250 \mathrm{MHz}): \delta(\mathrm{ppm})=$ 1.41-1.62 (m; $1 \mathrm{H}, \mathrm{CH}), 1.67-1.99(\mathrm{~m} ; 4 \mathrm{H}, \mathrm{CH}), 2.02\left(\mathrm{~s} ; 3 \mathrm{H}, \mathrm{N}-\mathrm{CH}_{3}\right), 2.12-$ 2.29 (m; $2 \mathrm{H}, \mathrm{CH}), 3.10-3.43(\mathrm{~m} ; 2 \mathrm{H}, \mathrm{CH}), 7.56-7.73$ (m; $2 \mathrm{H}$ aromat.), 7.97 (d; $\mathrm{J}=8.2 \mathrm{~Hz}, 1 \mathrm{H}$ aromat.), 8.64 (d; $\mathrm{J}=8.2 \mathrm{~Hz}, 1 \mathrm{H}$ aromat.), 8.73 (s; $1 \mathrm{H}$ aromat.), 9.14 (s; $1 \mathrm{H}$ aromat.).- MS: $\mathrm{m} / \mathrm{z}=226\left(16 \%, \mathrm{M}^{+\bullet}\right), 98(100$, $\left.\mathrm{C}_{6} \mathrm{H}_{12} \mathrm{~N}\right)^{+}$.

\section{4-(N-Formyl-pyrrolidin-2-yl)-isoquinoline (9a)}

To $100 \mathrm{mg}$ ( $0.51 \mathrm{mmole})$ of 3 in $2 \mathrm{ml}$ of dry $\mathrm{MeOH}$ were added in portions $38 \mathrm{mg}(1.0 \mathrm{mmole})$ of $\mathrm{NaBH}_{4}$ under $\mathrm{N}_{2}$ at $0^{\circ}$. After stirring for $3 \mathrm{~h}$ at $0^{\circ} 5 \mathrm{ml}$ of water were added, the mixture was made alkaline and extracted with $\mathrm{Et}_{2} \mathrm{O}$. The extracts were washed with brine, dried, and evaporated: brownish oil (5). Without purification raw 5 was treated with $3 \mathrm{ml}$ of acetic-formic anhydrid at $0^{\circ}$ for $2 \mathrm{~h}$. After addition of $5 \mathrm{ml}$ of ice water the mixture was made alkaline by $5 \% \mathrm{NaOH}$ and extracted with $\mathrm{CH}_{2} \mathrm{Cl}_{2}$. The extracts were washed with brine, dried, and evaporated to give a dark oil. Chromatography (MeOH:ethyl acetate $=8: 2)$ gave $76 \mathrm{mg}(66 \%)$ of $9 \mathrm{a}$ as a pale brown oil.- $\mathrm{C}_{14} \mathrm{H}_{14} \mathrm{~N}_{2} \mathrm{O}$ (226.3).- UV: $\lambda \max (\log \varepsilon)=217$ (4.35); 260 (3.52); 308 (3.26); $322 \mathrm{~nm}$ (3.35).- IR (film): $\widetilde{v}=2960(\mathrm{CH}) ; 1665 \mathrm{~cm}^{-1}$ (N-CHO).- ${ }^{1} \mathrm{H}-\mathrm{NMR}(250 \mathrm{MHz}): \delta(\mathrm{ppm})=1.91-2.20(\mathrm{~m} ; 3 \mathrm{H}, \mathrm{CH}), 2.46-$ $2.61(\mathrm{~m} ; 1 \mathrm{H}, \mathrm{CH}), 3.76-3.93(\mathrm{~m} ; 2 \mathrm{H}, \mathrm{CH}), 5.59-5.87(\mathrm{~m} ; 1 \mathrm{H}, \mathrm{CH}), 7.60-$ 8.07 (m; $4 \mathrm{H}$ aromat.), 8.25-8.50 (m; $2 \mathrm{H}, 1 \mathrm{H}$ aromat., $\mathrm{CHO}), 9.16-9.26(\mathrm{~m}$; $1 \mathrm{H}$ aromat.).- MS: $\mathrm{m} / \mathrm{z}=226\left(47 \%, \mathrm{M}^{+*}\right), 197(100, \mathrm{M}-\mathrm{CHO})^{+}$.

\section{4-(N-Formyl-piperidin-2-yl)-isoquinoline (10a)}

Following the procedure for $9 \mathrm{a}, 100 \mathrm{mg}(0.48 \mathrm{mmole})$ of 4 gave a brownish oil (6) which led to $55 \mathrm{mg}(48 \%) 10 \mathrm{a}$ as a light amber oil.$\mathrm{C}_{15} \mathrm{H}_{16} \mathrm{~N}_{2} \mathrm{O}$ (240.3).- UV: $\lambda \max (\log \varepsilon)=218$ (4.48); 272 (3.43); 308 (3.34); $321 \mathrm{~nm}$ (3.44).- IR (film): $\widetilde{v}=2960(\mathrm{CH}) ; 1665 \mathrm{~cm}^{-1}$ (N-CHO).${ }^{1} \mathrm{H}-\mathrm{NMR}(250 \mathrm{MHz}): \delta(\mathrm{ppm})=1.50-2.40(\mathrm{~m} ; 6 \mathrm{H}, \mathrm{CH}), 3.21-3.58(\mathrm{~m}$; $1.5 \mathrm{H}, \mathrm{CH}), 4.31-4.43(\mathrm{~m} ; 0.5 \mathrm{H}, \mathrm{CH}), 5.02-5.08(\mathrm{~m} ; 0.5 \mathrm{H}, \mathrm{CH}), 6.27-6.29$ $(\mathrm{m} ; 0.5 \mathrm{H}, \mathrm{CH}), 7.59-8.23(\mathrm{~m} ; 5 \mathrm{H}, 4 \mathrm{H}$ aromat., $\mathrm{CHO}), 8.56-8.60(2 \mathrm{~s} ; 1 \mathrm{H}$ aromat.), 9.18-9.25 (2s; $1 \mathrm{H}$ aromat.).- MS: $\mathrm{m} / \mathrm{z}=240\left(63 \%, \mathrm{M}^{+}\right), 211$ $\left(100, \mathrm{M}^{-} \mathrm{CHO}\right)^{+}$.

\section{4-(N-Formyl-pyrrolidin-2-yl)-1,2,3,4-tetrahydroisoquinoline (9)}

A paste of $50 \mathrm{mg}(0.22 \mathrm{mmole})$ of $9 \mathrm{a}$ and $25 \mathrm{mg}(0.4 \mathrm{mmole})$ of $\mathrm{NaBH}_{3} \mathrm{CN}$ was suspended and stirred in $2.5 \mathrm{ml}$ of a mixture of $\mathrm{CH}_{2} \mathrm{Cl}_{2}: \mathrm{HOAc}=10: 1$ for $24 \mathrm{~h}$ under $\mathrm{N}_{2}$ at room temp. Water was added, the mixture was made alkaline, and extracted with $\mathrm{CH}_{2} \mathrm{Cl}_{2}$. The extracts were washed with brine, dried, and evaporated to give a brownish oil. Chromatography (MeOH:ethyl acetate $=8: 2)$ gave $21 \mathrm{mg}(41 \%)$ of 9 as a pale brown oil.- $\mathrm{C}_{14} \mathrm{H}_{18} \mathrm{~N}_{2} \mathrm{O}$ (230.3).- UV: $\lambda \max (\log \varepsilon)=205$ (4.18), 259 nm (2.69).- IR (film): $\widetilde{v}=3300$ (NH); 2960 (CH); $1665 \mathrm{~cm}^{-1}$ (N-CHO).${ }^{1} \mathrm{H}-\mathrm{NMR}(250 \mathrm{MHz}): \delta(\mathrm{ppm})=1.39-2.28(\mathrm{~m} ; 5 \mathrm{H}, \mathrm{CH}), 2.61-2.80(\mathrm{~m} ; 1 \mathrm{H}$, $\mathrm{CH}), 2.88-3.19(\mathrm{~m} ; 2 \mathrm{H}, \mathrm{CH}), 3.22-3.84(\mathrm{~m} ; 2 \mathrm{H}, \mathrm{CH}), 4.05(\mathrm{~s} ; 1 \mathrm{H}, \mathrm{NH})$, 4.22-4.36 (m; $1 \mathrm{H}, \mathrm{CH}), 4.50-5.10(\mathrm{~m} ; 1 \mathrm{H}, \mathrm{CH}), 6.83-7.40(\mathrm{~m} ; 4 \mathrm{H}$ aromat.), 8.19-8.35 (m; $1 \mathrm{H}, \mathrm{CHO}) .-\mathrm{MS}: \mathrm{m} / \mathrm{z}=230\left(3 \%, \mathrm{M}^{+\bullet}\right), 70(100$, $\left.\mathrm{C}_{4} \mathrm{H}_{8} \mathrm{~N}\right)^{+}$.

\section{4-(N-Formyl-piperidin-2-yl)-1,2,3,4-tetrahydroisoquinoline (10)}

Following the procedure for $9,28 \mathrm{mg}(0.12 \mathrm{mmole})$ of $10 \mathrm{a}$ gave $8 \mathrm{mg}$ (27\%) 10 as a brown oil.- $\mathrm{C}_{15} \mathrm{H}_{20} \mathrm{~N}_{2} \mathrm{O}$ (244.3).- UV: $\lambda \max (\log \varepsilon)=202$ (4.20), $260 \mathrm{~nm}$ (2.71).- IR (film): $\tilde{\mathrm{v}}=3300(\mathrm{NH}) ; 2960(\mathrm{CH}) ; 1665 \mathrm{~cm}^{-1}$ (N-CHO).- MS: $\mathrm{m} / \mathrm{z}=244\left(9 \%, \mathrm{M}^{+\bullet}\right), 84\left(100, \mathrm{C}_{5} \mathrm{H}_{10} \mathrm{~N}\right)^{+}$.
6,7-Dimethoxy-1,4-dimethyl-4-(N-methylpyrrolidin-2-yl-)-3,4dihydroisoquinoline (11)

$100 \mathrm{mg}(0.31 \mathrm{mmole})$ acetamide $34 \mathrm{in} 1 \mathrm{ml}$ of absol. acetonitril were refluxed with $0.11 \mathrm{ml} \mathrm{POCl} 3$ for $3 \mathrm{~h}$ under $\mathrm{N}_{2}$. After cooling $\mathrm{POCl}_{3}$ and acetonitril were distilled off in vacuo, the residue was dissolved in $8 \mathrm{ml}$ of ice water, alkalized by $10 \% \mathrm{NaOH}$ and extracted with $\mathrm{CH}_{2} \mathrm{Cl}_{2}$. The org. phase was dried and evaporated: reddish mixture of diastereomers which was purified and separated by $\mathrm{cc}\left(\mathrm{CHCl}_{3}: \mathrm{EtOH}\right.$ :conc. $\left.\mathrm{NH}_{3}=85: 14: 1\right) ; 67$ mg (72\%), oil.- $\mathrm{C}_{18} \mathrm{H}_{26} \mathrm{~N}_{2} \mathrm{O}_{2}$ (302.2).- IR (film): $\widetilde{v}=2963(\mathrm{CH}) ; 2786(\mathrm{~N}-$ $\left.\mathrm{CH}_{3}\right) ; 1630 \mathrm{~cm}^{-1}(\mathrm{C}=\mathrm{N})$.- ${ }^{1} \mathrm{H}-\mathrm{NMR}: \delta(\mathrm{ppm})=1.0\left(\mathrm{~s} ; 3 \mathrm{H}, \mathrm{CH}_{3}\right) ; 1.3-1.6$ (m; $\left.4 \mathrm{H}, \mathrm{NCHCH}_{2} \underline{\mathrm{CH}}_{2}\right) ; 2.05-4.05\left(\mathrm{~m} ; 5 \mathrm{H}, 2 \times \mathrm{CH}_{2}, \mathrm{CH}\right) ; 2.15$ (s; 3H, =C$\left.\mathrm{CH}_{3}\right) ; 2.2\left(\mathrm{~s} ; 3 \mathrm{H}, \mathrm{N}_{-} \mathrm{CH}_{3}\right) ; 3.75$ and $3.8\left(2 \times \mathrm{s} ; 6 \mathrm{H}, \mathrm{OCH}_{3}\right) ; 6.75(\mathrm{~s} ; 1 \mathrm{H}$, arom.); 6.85 (s; $1 \mathrm{H}$, aromat.).- $\mathrm{MS}(70 \mathrm{eV}): \mathrm{m} / \mathrm{z}=219(3.5 \%, \mathrm{MH}-$ $\left.\mathrm{C}_{5} \mathrm{H}_{10} \mathrm{~N}\right)^{+\bullet}, 218\left(2.5, \mathrm{M}-\mathrm{C}_{5} \mathrm{H}_{10} \mathrm{~N}\right)^{+},\left(100, \mathrm{C}_{5} \mathrm{H}_{10}\right)^{+}$.- MS-FD: $\mathrm{m} / \mathrm{z}=303$ $(100 \%, \mathrm{MH})^{+}$.

\section{4-(I-N,N-Dimethylaminoethyl)-isoquinoline (17)}

To a solution of $65 \mathrm{ml}$ dimethylamine (5.6 $\mathrm{M}$ in absol. EtOH) were added $75 \mathrm{ml}$ of dry $\mathrm{EtOH}, 25 \mathrm{ml}$ of $5 \mathrm{~N}$ gaseous $\mathrm{HCl}$ in dry $\mathrm{EtOH}, 10.11 \mathrm{~g}$ (59 mmole) of 4-acetylisoquinoline $(36)^{9)}, 3.05 \mathrm{~g}$ (48.5 mmole) $\mathrm{NaBH}_{3} \mathrm{CN}$, and $10 \mathrm{~g}$ molecular sieve ( $3 \AA$ ). The mixture was stirred for 72 $\mathrm{h}$ at room temp. and acidified by $\mathrm{HCl}(\mathrm{pH}<2)$. After evaporation of excess $\mathrm{EtOH}$ the residue was suspended in water, alkalized with solid $\mathrm{KOH}(\mathrm{pH}>10)$, and brine was added. The solution was extracted with $\mathrm{Et}_{2} \mathrm{O}(3 \times 100 \mathrm{ml})$ and the extracts were washed with $10 \% \mathrm{NaHCO}_{3}$, dried, and evaporated to give a brown oil. Chromatography (MeOH:ethyl acetate $=1: 9$ ) and subsequent Kugelrohr distillation gave $4.8 \mathrm{~g}(41 \%) 17$ as a colourless oil. 17-monopicrate: needles, mp. $198^{\circ}$.- $\mathrm{C}_{19} \mathrm{H}_{19} \mathrm{~N}_{5} \mathrm{O}_{7}$ (429.4) Calcd. C 53.2 H 4.46 N 16.3 Found C 53.3 H 4.65 N 16.0.- UV: $\lambda$ max $(\log \varepsilon)=218$ (4.53); 272 (3.55); 3.09 (3.44); $322 \mathrm{~nm}$ (3.54).- IR (film): $\widetilde{v}=$ $2980 \mathrm{~cm}^{-1}(\mathrm{CH})$.- ${ }^{1} \mathrm{H}-\mathrm{NMR}$ (base): $\delta(\mathrm{ppm})=1.50(\mathrm{~d} ; \mathrm{J}=6.9 \mathrm{~Hz}, 3 \mathrm{H}, \mathrm{CH}-$ $\left.\mathrm{CH}_{3}\right), 2.30\left(\mathrm{~s} ; 6 \mathrm{H}, \mathrm{N}-\mathrm{CH}_{3}\right), 3.90\left(\mathrm{q} ; \mathrm{J}=6.9 \mathrm{~Hz}, 1 \mathrm{H}, \mathrm{C} \underline{\mathrm{H}}-\mathrm{CH}_{3}\right), 7.50-8.10$ (m; 3H aromat.), 8.50 (s; $1 \mathrm{H}$ aromat.), 8.60 (s; $1 \mathrm{H}$ aromat.), 9.20 (s; $1 \mathrm{H}$ aromat.).- MS: $\mathrm{m} / \mathrm{z}=200\left(20 \%, \mathrm{M}^{+\bullet}\right), 185\left(100, \mathrm{M}-\mathrm{CH}_{3}\right)^{+}$.

\section{2-Benzyl-4-(1-N,N-dimethylaminoethyl)-1,2,3,4-tetrahydroisoquinoline} (19)

Under $\mathrm{N}_{2} 0.10 \mathrm{~g}(0.2 \mathrm{mmole}) 44 \mathrm{in} 5 \mathrm{ml}$ of absol. THF were slowly added to $0.10 \mathrm{~g} \mathrm{LiAlH}_{4}$ in $10 \mathrm{ml}$ of absol. THF at $0^{\circ}$. After stirring for 15 $\min$ at $0^{\circ}$ and for $12 \mathrm{~h}$ at room temp., $10 \mathrm{ml}$ of THF:water $=1: 1$ were dropped into the suspension with cooling, keeping the temp. at $0^{\circ}$. After stirring for $5 \mathrm{~min}$ the mixture was extracted $3 \mathrm{x}$ with $5 \mathrm{ml}$ of $\mathrm{Et}_{2} \mathrm{O}$ each. The org. phase was washed with brine and water, dried, and evaporated: dark brown oil, purified by cc (ethyl acetate: $\mathrm{MeOH}=1: 1$ ): $31 \mathrm{mg}$ (34\%) yellow oil.- UV: $\lambda \max (\log \varepsilon)=207 \mathrm{~nm}$ (4.62).- IR (film): $\widetilde{v}=2931(\mathrm{CH}), 2965$ $\mathrm{cm}^{-1}(\mathrm{CH}) .{ }^{1} \mathrm{H}-\mathrm{NMR}(250 \mathrm{MHz}): \delta(\mathrm{ppm})=0.81(\mathrm{~d} ; \mathrm{J}=5.4 \mathrm{~Hz}, 3 \mathrm{H}, \mathrm{CH}-$ $\left.\mathrm{CH}_{3}\right), 2.18\left(\mathrm{~s} ; 6 \mathrm{H}, \mathrm{N}\left(\mathrm{CH}_{3}\right)_{2}\right), 2.37\left(\mathrm{dd} ; \mathrm{J}_{1}=2.6 \mathrm{~Hz}, \mathrm{~J}_{2}=7.7 \mathrm{~Hz}, 1 \mathrm{H}, \mathrm{N}-\right.$ $\left.\mathrm{CH}_{2}-\mathrm{CH}\right), 2.64-2.72\left(\mathrm{~m} ; 1 \mathrm{H}, \mathrm{CH}-\mathrm{C} \underline{\mathrm{H}}-\mathrm{CH}_{3}\right), 2.88-3.06$ (m; $1 \mathrm{H}, \mathrm{C} \underline{\mathrm{H}}-\mathrm{CH}-$ $\mathrm{CH}_{3}$ ), $3.32\left(\mathrm{~d} ; 1 \mathrm{H}, \mathrm{J}_{1}=2.6 \mathrm{~Hz}, \mathrm{~J}_{2}=7.7 \mathrm{~Hz}, \mathrm{~N}-\mathrm{CH}_{2}-\mathrm{CH}\right.$ ), 3.37 (AB-system; $\mathrm{J}=14.7 \mathrm{~Hz}, 1 \mathrm{H}, \mathrm{Ar}-\mathrm{C}_{2}-\mathrm{N}$ ), 3.66 (s, $2 \mathrm{H}, \mathrm{CH}_{2}-\mathrm{Ph}$ ), 3.85 (AB-system; $\mathrm{J}=$ 14.7 Hz, $\left.1 \mathrm{H}, \mathrm{Ar}-\underline{\mathrm{C}}_{2}-\mathrm{N}\right), 6.91-7.01$ (m; $1 \mathrm{H}$ aromat.), 7.07-7.21 (m; $3 \mathrm{H}$ aromat.), 7.28-7.42 (m; $5 \mathrm{H}$ aromat.).- $\mathrm{MS}(70 \mathrm{eV}): \mathrm{m} / \mathrm{z}=294\left(0.5 \%, \mathrm{M}^{+^{*}}\right)$, $250\left(0.5, \mathrm{M}-\mathrm{NMe}_{2}\right)^{+}, 222\left(0.5, \mathrm{M}-\mathrm{CHMeNMe}_{2}\right)^{+}, 91\left(10, \mathrm{C}_{7} \mathrm{H}_{7}\right)^{+}, 72$ $\left(100, \mathrm{CHMeNMe}_{2}\right)^{+}$.

4-(1-Aminoethyl)-6-methoxy-1,4-dimethyl-1,2,3,4-tetrahydroisoquinoline (20)

$0.050 \mathrm{~g}$ (0.19 mmole) 56 were dissolved in $1 \mathrm{ml}$ of absol. THF/1,2dimethoxyethane $1: 1$, mixed with $1 \mathrm{ml}$ of $\mathrm{BH}_{3}$-THF-complex, and stirred for $15 \mathrm{~min}$ at room temp., for $1 \mathrm{~h}$ at $65^{\circ}$, and for $5 \mathrm{~h}$ at $105^{\circ}$. After addition 
of $2 \mathrm{ml}$ of EtOH and $1 \mathrm{ml}$ of water at $0^{\circ}$, alkalization by dil. $\mathrm{NH}_{3}$, extraction with $\mathrm{Et}_{2} \mathrm{O}$, and drying, the solvents were distilled off in vacuo (Kugelrohr, 0.02 torr, $\left.50^{\circ}\right)$. Amine 20 is purified by prep. tlc $\left(\mathrm{CH}_{2} \mathrm{Cl}_{2}\right.$ :acetone: $\mathrm{MeOH}=6: 3: 1): 35 \mathrm{mg}(78 \%)$ colourless oil, which could not be further purified.- UV: $\lambda \max (\log \varepsilon)=203(4.24), 250(3.20), 278 \mathrm{~nm}$ (2.75).- IR (film): $\tilde{\mathrm{v}}=3332(\mathrm{NH}), 2925 \mathrm{~cm}^{-1}(\mathrm{CH}) .{ }^{1} \mathrm{H}-\mathrm{NMR}: \delta(\mathrm{ppm})=$ 1.40 (d; J = 8.2 Hz, 3H, CH-C $\left.\underline{\mathrm{H}}_{3}\right), 1.51$ (d; J = 6.4 Hz, 3H, CH-C $\left.\underline{\mathrm{H}}_{3}\right), 1.90$ (s; $3 \mathrm{H}, \mathrm{CH}_{3}$ ), 2.25 (s; 3H, NH, $\mathrm{NH}_{2}$, exchangeable), 3.02 (AB-system, $\mathrm{J}=$ $9.3 \mathrm{~Hz}, 1 \mathrm{H}, \underline{\mathrm{HCH}}$ ), 3.18 (AB-system, $\mathrm{J}=9.3 \mathrm{~Hz}, 1 \mathrm{H}, \mathrm{HC} \underline{\mathrm{H}}), 3.75$ (q; J = $\left.8.2 \mathrm{~Hz}, 1 \mathrm{H}, \mathrm{CHCH}_{3}\right), 3.88\left(\mathrm{~s} ; 3 \mathrm{H}, \mathrm{OCH}_{3}\right), 4.10(\mathrm{q} ; \mathrm{J}=6.4 \mathrm{~Hz}, 1 \mathrm{H}$, $\left.\mathrm{CHCH}_{3}\right), 6.68-7.40$ ( $\mathrm{m} ; 3 \mathrm{H}$ aromat.).- $\mathrm{MS}(70 \mathrm{eV}): \mathrm{m} / \mathrm{z}=234\left(1.7 \%, \mathrm{M}^{+\cdot}\right)$, $233(6, \mathrm{M}-\mathrm{H})^{+}, 219\left(20, \mathrm{M}-\mathrm{CH}_{3}\right)^{+}, 218\left(87,233-\mathrm{CH}_{3}\right)^{+\bullet}, 205(8, \mathrm{RDA})$, $190\left(33, \mathrm{M}-\mathrm{H}_{3} \mathrm{CCHNH}_{2}\right)^{+}$.

\section{2-(3,4-Dimethoxyphenyl)-2-(N-methylpyrrol-2-yl)-1-nitropropane (28)} and 1-(3,4-Dimethoxyphenyl)-1-(N-methylpyrrol-2-yl)-2-nitropropane (29)

The solution of $10.9 \mathrm{mg}$ (0.38 mmole) 2-(3,4-dimethoxyphenyl)-2-( $N$ methylpyrrol-2-yl)-1-nitroethane (27) ${ }^{2)}$ in $5 \mathrm{ml}$ of absol. THF and $0.70 \mathrm{ml}$ DMPU was cooled to $-80^{\circ}$. Then $1.20 \mathrm{ml} \mathrm{n}$-BuLi (15\% in hexane) were added, resulting in a red-brown solution. After stirring for $30 \mathrm{~min}$ at $-80^{\circ}$ $0.60 \mathrm{ml} \mathrm{CH}_{3} \mathrm{I}$ were added drop by drop, then the cooling device was removed. After $3 \mathrm{~h}$, when the mixture has reached room temp., it was stored at $+4^{\circ} \mathrm{C}$ for $24 \mathrm{~h}$, then it was cooled to $-50^{\circ}$, and 8-10 drops of glacial acetic acid were added. After addition of $2 \mathrm{ml}$ of water and $30 \mathrm{ml}$ of $\mathrm{Et}_{2} \mathrm{O}$, the mixture was allowed to warm up to room temp. Then the org. phase was washed with a satd. solution of $\mathrm{NaHCO}_{3}$ and with water, dried, and the solvents were evaporated. Purification by $\mathrm{cc}\left(\mathrm{CH}_{2} \mathrm{Cl}_{2}\right): 98 \mathrm{mg}$ of a mixture: $36 \% 27 ; 41 \%$ 28; $22 \%$ 29.- ${ }^{1} \mathrm{H}-\mathrm{NMR}: \delta(\mathrm{ppm})=1.69\left(\mathrm{~d} ; 0.6 \mathrm{H} \mathrm{CHCH}{ }_{3}\right.$, 29), $1.91\left(\mathrm{~s} ; 1.23 \mathrm{H}, \mathrm{CCH}_{3}, 28\right), 3.10\left(\mathrm{~s} ; 0.41 \mathrm{H}, \mathrm{NCH}_{3}\right), 3.35(\mathrm{~s} ; 0.36 \mathrm{H}$, $\left.\mathrm{NCH}_{3}\right), 3.45\left(\mathrm{~s} ; 0.22 \mathrm{H}, \mathrm{NCH}_{3}\right) ; 3.70-3.91\left(\mathrm{~m} ; 6 \mathrm{H}, \mathrm{OCH}_{3}\right) ; 4.12-5.25(\mathrm{~m}$; 2.34H, $\mathrm{CHCH}_{2}$ and $\mathrm{CCH}_{2}$ ), 6.03-6.92 (m; $6 \mathrm{H}$ aromat.).

\section{2-(3,4-Dimethoxyphenyl)-2-(N-methylpyrrol-2-yl)-acetonitril (30)}

To the solution of $2.9 \mathrm{~g}(10.0 \mathrm{mmole}) 27^{2)}$ in $10 \mathrm{ml}$ of absol. 1,2-dichloroethane were added $4.60 \mathrm{ml}$ ( $25.0 \mathrm{mmole})$ tris-(dimethylamino)phosphine within 5 min under $\mathrm{N}_{2}$. The solution was slowly warmed to $50^{\circ}$ and then refluxed for 20 min under $\mathrm{N}_{2}$. After cooling $20 \mathrm{ml}$ of water were added and the aqueous phase was extracted $2 \mathrm{x}$ with $25 \mathrm{ml}$ of $\mathrm{CH}_{2} \mathrm{Cl}_{2}$ each. The org. phase was washed with water $(2 \mathrm{x})$ and satd. $\mathrm{NaCl}$ solution, dried, and evaporated at $40^{\circ}$ : yellow oil, purification by $\mathrm{cc}\left(\mathrm{CH}_{2} \mathrm{Cl}_{2}\right): 1.0 \mathrm{~g} 30$ (40\%).- $\mathrm{C}_{16} \mathrm{H}_{16} \mathrm{~N}_{2} \mathrm{O}_{2}$ (256.1).- IR (film): $\widetilde{\mathrm{v}}=2938(\mathrm{CH}) ; 2838\left(\mathrm{~N}-\mathrm{CH}_{3}\right)$; $2242(\mathrm{CN}) ; 1595 \mathrm{~cm}^{-1} .{ }^{1} \mathrm{H}-\mathrm{NMR}: \delta(\mathrm{ppm})=3.4\left(\mathrm{~s} ; 3 \mathrm{H}, \mathrm{NCH}_{3}\right), 3.8(\mathrm{~s} ;$ $\left.3 \mathrm{H}, \mathrm{OCH}_{3}\right), 3.85\left(\mathrm{~s} ; 3 \mathrm{H}, \mathrm{OCH}_{3}\right), 5.15(\mathrm{~s} ; 1 \mathrm{H}, \mathrm{CHCN}), 6.0-6.9(\mathrm{~m} ; 6 \mathrm{H}$ aromat.).- MS (70 eV): $\mathrm{m} / \mathrm{z}=256\left(98 \%, \mathrm{M}^{+*}\right), 241\left(28, \mathrm{M}-\mathrm{CH}_{3}\right)^{+}, 230(20$, $\mathrm{M}-\mathrm{CN})^{+}, 225\left(100, \mathrm{M}-\mathrm{OCH}_{3}\right)^{+}$.

\section{2-(3,4-Dimethoxyphenyl)-2-(N-methylpyrrol-2-yl)-propionitril (31)}

Under $\mathrm{N}_{2} 8 \mathrm{ml}$ of absol. THF and absol. $\mathrm{Et}_{2} \mathrm{O}$ each were cooled to $-70^{\circ}$ in a three-necked flask with stirrer and thermometer. Then $2.70 \mathrm{ml} \mathrm{n}$-BuLi (15\% in hexane) were added slowly under $\mathrm{N}_{2}$ followed by dropwise addition of $0.512 \mathrm{~g}$ ( $2.0 \mathrm{mmole})$ of nitril $30 \mathrm{in} 5.3 \mathrm{ml}$ of absol. THF. After removing of the cooling bath the mixture was allowed to warm up to $-30^{\circ}$ under stirring. At this temp. $0.9 \mathrm{~g}$ of $\mathrm{CH}_{3} \mathrm{I}$ in $20 \mathrm{ml}$ of absol. $\mathrm{Et}_{2} \mathrm{O}$ were added in one portion (during this addition the temp. may rise to $-10^{\circ}$ ). The mixture was stirred at room temp. for $20 \mathrm{~h}$ under $\mathrm{N}_{2}$, then cooled to $-70^{\circ}$ again, and $5 \mathrm{ml}$ of $\mathrm{EtOH}$ and $5 \mathrm{ml}$ of satd. $\mathrm{NH}_{4} \mathrm{Cl}$ solution were added. The cooling device was removed and the mixture was stirred until $\mathrm{NH}_{4} \mathrm{Cl}$ was dissolved. The brownish solution was mixed with $5 \mathrm{ml}$ of satd. $\mathrm{NaCl}$ solution and extracted $3 \mathrm{x}$ with $5 \mathrm{ml}$ of $\mathrm{Et}_{2} \mathrm{O}$ each. The org. phase was washed with water, dried, evaporated, and purified by $\mathrm{cc}\left(\mathrm{CH}_{2} \mathrm{Cl}_{2}\right): 412$ mg 31 (76\%), colourless crystals, m.p. 85-87 (hexane:EtOH = 9:1).$\mathrm{C}_{16} \mathrm{H}_{18} \mathrm{~N}_{2} \mathrm{O}_{2}$ (270.1) Calcd. C $71.0 \mathrm{H} 6.66 \mathrm{~N} 10.3$ Found C $71.0 \mathrm{H} 6.80 \mathrm{~N}$ 10.1.- IR (film): $\tilde{\mathrm{v}}=2938(\mathrm{CH}) ; 2838\left(\mathrm{~N}_{-} \mathrm{CH}_{3}\right) ; 2234(\mathrm{CN}) ; 1593 \mathrm{~cm}^{-1}$ $(\mathrm{C}=\mathrm{C})$.- ${ }^{1} \mathrm{H}-\mathrm{NMR}: \delta(\mathrm{ppm})=1.95\left(\mathrm{~s} ; 3 \mathrm{H}, \mathrm{CH}_{3}\right) ; 3.25\left(\mathrm{~s} ; 3 \mathrm{H}, \mathrm{NCH}_{3}\right) ; 3.75$ and $3.82\left(2 \times \mathrm{s} ; 6 \mathrm{H}, \mathrm{OCH}_{3}\right) ; 6.0-6.82(\mathrm{~m} ; 6 \mathrm{H}$ aromat.). $\mathrm{MS}(70 \mathrm{eV}): \mathrm{m} / \mathrm{z}=$ $270\left(32 \%, \mathrm{M}^{+\bullet}\right), 255\left(100, \mathrm{M}-\mathrm{CH}_{3}\right)^{+}$.

\section{2-(3,4-Dimethoxyphenyl)-2-(N-methylpyrrol-2-yl)-propylamine (32)}

Method A: $20 \mathrm{~g}$ Raney-Ni were activated and suspended in a solution of $3.1 \mathrm{~g}(11.5 \mathrm{mmole})$ nitril $31 \mathrm{in} 20 \mathrm{ml}$ of absol. EtOH and $3 \mathrm{ml}$ of $\mathrm{EtOH}$, satd. with $\mathrm{NH}_{3}$ gas. This mixture was stirred for $7 \mathrm{~d}$ under $\mathrm{H}_{2}$ of 14 bar. Stirring was continued with $10 \mathrm{~g}$ activated Raney-Ni for $5 \mathrm{~d}$. After filtration and evaporation the residue was dissolved in $\mathrm{Et}_{2} \mathrm{O}$ and the solution was washed with brine. The aqueous phase was thorougly extracted with $\mathrm{Et}_{2} \mathrm{O}$, the ether phase was dried and evaporated. Purification by $\mathrm{cc}$ $\left(\mathrm{CH}_{2} \mathrm{Cl}_{2}: \mathrm{MeOH}=9: 1\right): 2.67 \mathrm{~g} 32(85 \%)$, yellowish oil.- $\mathrm{C}_{16} \mathrm{H}_{22} \mathrm{~N}_{2} \mathrm{O}_{2}$ (274.2).- MS (70 eV): $\mathrm{m} / \mathrm{z}=274\left(1.4 \%, \mathrm{M}^{+}\right), 244\left(100, \mathrm{M}-\mathrm{CH}_{2} \mathrm{NH}_{2}\right)^{+}$.

Method B: $300 \mathrm{mg}$ (1.11 mmole) 31 were dried over night at room temp., 0.01 Torr, dissolved in $5 \mathrm{ml}$ of absol. THF, and added slowly to a

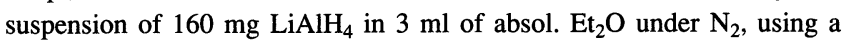
syringe and a diaphragma. Then the mixture was heated to reflux for $5 \mathrm{~h}$ under $\mathrm{N}_{2}$, cooled to $0^{\circ}$ and quenched by careful addition of a THF/ice mixture. After $15 \mathrm{~min} \mathrm{Al}(\mathrm{OH})_{3}$ was separated, the aqueous phase was extracted $2 \mathrm{x}$ with $\mathrm{Et}_{2} \mathrm{O}$, the combined org. phase was dried and evaporated: yellowish oil; for purification see above: $270 \mathrm{mg}$ (90\%) 32, which was directly acetylated to 33 .

\section{$N$-Acetyl-2-(3,4-dimethoxyphenyl)-2-(N-methylpyrrol-2-yl)-propylamine} (33)

Under $\mathrm{N}_{2} 1.20 \mathrm{~g}$ (4.44 mmole) 32 were dissolved in $20 \mathrm{ml}$ of acetic acid anhydride under cooling, then slowly warmed to $50^{\circ}$ and stirred at this temp. for 2 h.- After cooling to $0^{\circ}$, hydrolysis, addition of $\mathrm{NaOH}$ to $\mathrm{pH} 8$ 9, separate extractions with $\mathrm{Et}_{2} \mathrm{O}$ and $\mathrm{CH}_{2} \mathrm{Cl}_{2}$, the org. phase was dried and evaporated. Purification by cc (ethyl acetate): $1.19 \mathrm{~g}$ (85\%), m.p. $95-97^{\circ}$ (EtOH).- $\mathrm{C}_{18} \mathrm{H}_{24} \mathrm{~N}_{2} \mathrm{O}_{3}$ (316.2) Calcd. C 68.3 H 7.7 N 8.9 Found C $67.9 \mathrm{H}$ $8.1 \mathrm{~N} 7.6$, deviations in elem. analysis are due to EtOH ( $\left.{ }^{1} \mathrm{H}-\mathrm{NMR}\right)$.- IR (film): $\widetilde{v}=3370(\mathrm{NH}) ; 2963(\mathrm{CH}) ; 1655 \mathrm{~cm}^{-1}(\mathrm{CO}) .{ }^{1} \mathrm{H}-\mathrm{NMR}: \delta(\mathrm{ppm})=$ $1.5\left(\mathrm{~s} ; 3 \mathrm{H}, \mathrm{CH}_{3}\right) ; 1.8\left(\mathrm{~s} ; 3 \mathrm{H}, \mathrm{COCH}_{3}\right) ; 2.95\left(\mathrm{~s} ; 3 \mathrm{H}, \mathrm{NCH}_{3}\right) ; 3.6$ and $3.7(2 \mathrm{x}$ s; $\left.6 \mathrm{H}, \mathrm{OCH}_{3}\right) ; 5.0-5.4$ (br. m; $\left.1 \mathrm{H}, \mathrm{NH}\right) ; 5.95-6.7$ (m; $6 \mathrm{H}$ aromat.).- $\mathrm{MS}(70$ $\mathrm{eV}): \mathrm{m} / \mathrm{z}=257\left(6 \%, \mathrm{M}-\mathrm{CH}_{3} \mathrm{CONH}_{2}\right)^{+}, 244\left(100, \mathrm{M}-\mathrm{CH}_{2} \mathrm{NHCOCH}_{3}\right)^{+}$.

\section{$N$-Acetyl-2-(3,4-dimethoxyphenyl)-2-(N-methylpyrrolidin-2-yl)- propylamine (34)}

The solution of $2.20 \mathrm{~g}$ (6.96 mmole) 33 in $18 \mathrm{ml}$ of absol. acetic acid was stirred vigorously with $700 \mathrm{mg} \mathrm{Rh} / \mathrm{C}$ under $\mathrm{H}_{2}$ (25 bar) for $5 \mathrm{~d}$.- After cooling the solution was neutralized with $10 \% \mathrm{NaOH}$, alkalized with $\mathrm{NaHCO}_{3}$, and filtrated. The catalyst was washed with $\mathrm{CH}_{2} \mathrm{Cl}_{2}$ and the aqueous phase was extracted with $\mathrm{CH}_{2} \mathrm{Cl}_{2}$. The org. layer was dried and evaporated. Purification by $\mathrm{cc}\left(\mathrm{CH}_{2} \mathrm{Cl}_{2}: \mathrm{MeOH}=4: 1\right)$ : brownish oil, crystallizing from diisopropyl ether:acetone $=10: 1$ : colourless crystals, 1.47 g (66\%), m.p. 84-88 ${ }^{\circ}$ - $\mathrm{C}_{18} \mathrm{H}_{28} \mathrm{~N}_{2} \mathrm{O}_{3}$ (320.2) Calcd. C $67.4 \mathrm{H} 8.75 \mathrm{~N}$ 8.5 Found C 66.7 H 8.19 N 9.1.- IR (film): $\widetilde{v}=3372(\mathrm{NH}) ; 2963(\mathrm{CH})$; $2786\left(\mathrm{NCH}_{3}\right) ; 1660 \mathrm{~cm}^{-1}(\mathrm{CO}) .-{ }^{1} \mathrm{H}-\mathrm{NMR}: \delta(\mathrm{ppm})=1.3\left(\mathrm{~s} ; 3 \mathrm{H}, \mathrm{CH}_{3}\right)$; $1.6-1.9\left(\mathrm{~m} ; 2 \mathrm{H}, \mathrm{CH}_{2}\right) ; 1.95\left(\mathrm{~s} ; 3 \mathrm{H}, \mathrm{COCH}_{3}\right) ; 2.15\left(\mathrm{~s} ; 3 \mathrm{H}, \mathrm{NCH}_{3}\right) ; 2.35-4.3$ (m; 7H, $\left.3 \times \mathrm{CH}_{2}, \mathrm{CH}\right) ; 3.95$ (s; $\left.6 \mathrm{H}, \mathrm{OCH}_{3}\right)$; 6.9-7.1 (m; $3 \mathrm{H}$ aromat.), 7.5$7.8(\mathrm{~m} ; 1 \mathrm{H}, \mathrm{NH})$. $^{\mathrm{MS}}(70 \mathrm{eV}): \mathrm{m} / \mathrm{z}=320\left(0.12 \%, \mathrm{M}^{+\bullet}\right), 248(0.06, \mathrm{M}-$ $\left.\mathrm{CH}_{2} \mathrm{NHCOCH}_{3}\right)^{+}, 84\left(100, \mathrm{C}_{5} \mathrm{H}_{10} \mathrm{~N}\right)^{+}$.- MS-FD: $\mathrm{m} / \mathrm{z}=321(100 \%, \mathrm{MH})^{+}$.

\section{4-(1-N-Methylaminoethyl)-isoquinoline (37)}

Following the procedure for $17,9 \mathrm{ml}$ of $8 \mathrm{M}$ methylamine in absol. $\mathrm{EtOH}, 16 \mathrm{ml}$ of EtOH, $5.6 \mathrm{ml}$ of $5 \mathrm{~N} \mathrm{HCl}$ in $\mathrm{EtOH}, 2 \mathrm{~g}$ of $36^{9)}, 0.5 \mathrm{~g}(7.9$ 
mmole) of $\mathrm{NaBH}_{3} \mathrm{CN}$ and $2 \mathrm{~g}$ molecular sieve ( $3 \AA$ ) gave $1.04 \mathrm{~g}(48 \%) 37$ as a colourless oil.- $\mathrm{C}_{12} \mathrm{H}_{14} \mathrm{~N}_{2}$ (186.3).- UV: $\lambda \max (\log \varepsilon)=218(4.43)$; 272 (3.56); 309 (3.44); $322 \mathrm{~nm}$ (3.55).- IR (film): $\widetilde{v}=3300$ (NH); 2980 $\mathrm{cm}^{-1}(\mathrm{CH}) .-{ }^{1} \mathrm{H}-\mathrm{NMR}: \delta(\mathrm{ppm})=1.50-1.55\left(\mathrm{~d} ; \mathrm{J}=6.9 \mathrm{~Hz}, 3 \mathrm{H}, \mathrm{CH}-\mathrm{CH}_{3}\right)$, $1.80(\mathrm{~s} ; 1 \mathrm{H}, \mathrm{NH}), 2.40\left(\mathrm{~s} ; 3 \mathrm{H}, \mathrm{NCH}_{3}\right), 4.30-4.55(\mathrm{q} ; \mathrm{J}=6.9 \mathrm{~Hz}, 1 \mathrm{H}, \mathrm{C} \underline{\mathrm{H}}-$ $\mathrm{CH}_{3}$ ), 7.25-8.40 (m; $4 \mathrm{H}$ aromat.), 8.70 (s; $1 \mathrm{H}$ aromat.), 9.20 (s; $1 \mathrm{H}$ aromat.).- MS: $\mathrm{m} / \mathrm{z}=186\left(8 \%, \mathrm{M}^{+*}\right), 171\left(100, \mathrm{M}-\mathrm{CH}_{3}\right)^{+}$.

\section{4-(1-N-Formyl-N-methylaminoethyl)-isoquinoline (38)}

$355 \mathrm{mg}$ (1.9 mmole) of 37 were treated with $5 \mathrm{ml}$ of acetic-formic anhydride at $0^{\circ}$ for $15 \mathrm{~min}$. After addition of $10 \mathrm{ml}$ of ice water the mixture was made alkaline by $5 \% \mathrm{NaOH}$ and extracted with $\mathrm{Et}_{2} \mathrm{O}$. The extracts were washed with brine, dried, and evaporated. Kugelrohr distillation gave 385 $\mathrm{mg}(95 \%) 38$ as a colourless oil.- 38-monopicrate: needles, m.p. $194^{\circ}$.$\mathrm{C}_{19} \mathrm{H}_{17} \mathrm{~N}_{5} \mathrm{O}_{8}$ (443.4) Calcd. C $51.4 \mathrm{H} 3.86 \mathrm{~N} 15.8$ Found C $50.8 \mathrm{H} 3.86 \mathrm{~N}$ 15.6.- UV: $\lambda \max (\log \varepsilon)=220$ (4.41); 271 (3.78); 307 (3.60); $320 \mathrm{~nm}$ (3.73).- IR (film): $\tilde{v}=2990(\mathrm{CH}) ; 1665 \mathrm{~cm}^{-1}$ (N-CHO).- ${ }^{1} \mathrm{H}-\mathrm{NMR}(250$ $\mathrm{MHz}$ ) (base): $\delta(\mathrm{ppm})=1.71-1.86\left(2 \mathrm{~d} ; \mathrm{J}=6.9 \mathrm{~Hz}, 3 \mathrm{H}, \mathrm{CH}-\mathrm{CH}_{3}\right), 2.53-$ $2.68\left(2 \mathrm{~s} ; 3 \mathrm{H}, \mathrm{NCH}_{3}\right), 5.46-5.49\left(\mathrm{q} ; \mathrm{J}=6.9 \mathrm{~Hz}, 0.2 \mathrm{H}, \mathrm{C} \underline{\mathrm{H}}-\mathrm{CH}_{3}\right), 6.37-6.45$ (q; J $\left.=6.9 \mathrm{~Hz}, 0.8 \mathrm{H}, \mathrm{C} \underline{\mathrm{H}}-\mathrm{CH}_{3}\right), 7.62-8.12(\mathrm{~m} ; 5 \mathrm{H}, 4 \mathrm{H}$ aromat., $\mathrm{CHO}$ ), 8.57-8.59 (2s; $1 \mathrm{H}$ aromat.), 9.24-9.26 (2s; $1 \mathrm{H}$ aromat.).- MS: $\mathrm{m} / \mathrm{z}=214$ $\left(100 \%, \mathrm{M}^{+*}\right)$.

\section{2-Formyl-4-(1-N-methylaminoethyl)-1,2,3,4-tetrahydroisoquinoline (41)}

A paste of $250 \mathrm{mg}$ (1.2 mmole) 38 and $125 \mathrm{mg}(2 \mathrm{mmole})$ of $\mathrm{NaBH}_{3} \mathrm{CN}$ was suspended and stirred in $11 \mathrm{ml}$ of $\mathrm{CH}_{2} \mathrm{Cl}_{2}: \mathrm{HOAc}=10: 1$ for $24 \mathrm{~h}$ under $\mathrm{N}_{2}$ at room temp. Water was added, the mixture was made alkaline and extracted with $\mathrm{CH}_{2} \mathrm{Cl}_{2}$. The extracts were washed with brine, dried, and evaporated to give a yellowish oil. Chromatography $\left(\mathrm{MeOH}: \mathrm{CH}_{2} \mathrm{Cl}_{2}=\right.$ 3:7) gave $165 \mathrm{mg}(62 \%) 41$ as a pale yellow oil.- $\mathrm{C}_{13} \mathrm{H}_{18} \mathrm{~N}_{2} \mathrm{O}$ (218.3).UV: $\lambda \max (\log \varepsilon)=205$ (3.95), 260 (2.45).- IR (film): $\widetilde{\mathrm{v}}=3300(\mathrm{NH})$; $2970(\mathrm{CH}) ; 1665 \mathrm{~cm}^{-1}$ (N-CHO).- ${ }^{1} \mathrm{H}-\mathrm{NMR}(250 \mathrm{MHz}): \delta(\mathrm{ppm})=0.97-$ $1.27(\mathrm{~m} ; 3 \mathrm{H}, \mathrm{CH}), 1.73(\mathrm{~s} ; 1 \mathrm{H}, \mathrm{NH}), 2.38-2.43(\mathrm{~m} ; 2 \mathrm{H}, \mathrm{CH}), 2.58-3.55(\mathrm{~m}$; $4 \mathrm{H}, \mathrm{CH}), 4.04-5.00(\mathrm{~m} ; 3 \mathrm{H}, \mathrm{CH}), 7.03-7.31(\mathrm{~m} ; 4 \mathrm{H}$ aromat. $), 8.14-8.30$ (m; $1 \mathrm{H}, \mathrm{CHO})$.- MS: $\mathrm{m} / \mathrm{z}=218\left(2 \%, \mathrm{M}^{+\cdot}\right), 58\left(100, \mathrm{C}_{3} \mathrm{H}_{8} \mathrm{~N}\right)^{+}$.

\section{3,4-Dimethyl-benzo[f]-1,3-diaza-bicyclo[3.3.1]nonane (42)}

$110 \mathrm{mg}(0.5 \mathrm{mmole})$ of $\mathbf{4 1}$ in $2 \mathrm{ml}$ of dry $\mathrm{Et}_{2} \mathrm{O}$ were added dropwise to $95 \mathrm{mg}$ ( $2.5 \mathrm{mmole})$ of $\mathrm{LiAlH}_{4}$ in $3 \mathrm{ml}$ of $\mathrm{Et}_{2} \mathrm{O}$. The mixture was refluxed for $2 \mathrm{~h}$. Excess $\mathrm{LiAlH}_{4}$ was destroyed by careful addition of $\mathrm{MeOH}$ under cooling. After addition of $3 \mathrm{ml}$ of ice water the mixture was extracted with $\mathrm{Et}_{2} \mathrm{O}(3 \times 10 \mathrm{ml})$ and the extracts were washed with $10 \% \mathrm{NaHCO}_{3}$, dried, and evaporated to give a brown oil. Chromatography $\left(\mathrm{MeOH}: \mathrm{CH}_{2} \mathrm{Cl}_{2}=\right.$ 2:8) gave $48 \mathrm{mg}(47 \%)$ of 42 as an amber oil.- $\mathrm{C}_{13} \mathrm{H}_{18} \mathrm{~N}_{2}$ (202.3).- IR (film): $\widetilde{v}=2970 \mathrm{~cm}^{-1}(\mathrm{CH}) .{ }^{1} \mathrm{H}-\mathrm{NMR}: \delta(\mathrm{ppm})=1.15-1.25(\mathrm{~m} ; 3 \mathrm{H}, \mathrm{CH})$, 2.05 (s; $\left.3 \mathrm{H}, \mathrm{NCH}_{3}\right), 2.25-2.30(\mathrm{~m} ; 1 \mathrm{H}, \mathrm{CH}), 2.40-2.50(\mathrm{~m} ; 1 \mathrm{H}, \mathrm{CH}), 2.65-$ 2.85 (m; $1 \mathrm{H}, \mathrm{CH}), 2.90-3.05(\mathrm{~m} ; 1 \mathrm{H}, \mathrm{CH}), 3.20-3.60(\mathrm{~m} ; 2 \mathrm{H}, \mathrm{CH}), 3.75-$ $3.90(\mathrm{~m} ; 1 \mathrm{H}, \mathrm{CH}), 4.05-4.50(\mathrm{~m} ; 1 \mathrm{H}, \mathrm{CH}), 7.00-7.35$ (m; $4 \mathrm{H}$ aromat.).MS: $\mathrm{m} / \mathrm{z}=202\left(7 \%, \mathrm{M}^{+\cdot}\right), 145\left(100, \mathrm{M}-\mathrm{C}_{3} \mathrm{H}_{7} \mathrm{~N}\right)^{+}$.

\section{2-Benzyl-4-[1-(N-formyl-N-methylamino)-ethyl]isoquinolinium bromide} (43)

Under $\mathrm{N}_{2} 1.50 \mathrm{~g}$ (8.79 mmole, $\left.300 \%\right)$ benzylbromide were added dropwise at $0^{\circ}$ during $2 \mathrm{~h}$ to $0.63 \mathrm{~g}$ (2.93 mmole) formylamide 38 , dissolved in $5 \mathrm{ml}$ of absol. benzene. The mixture was stirred for $2 \mathrm{~h}$ at room temp. and refluxed for $10 \mathrm{~h}$. A brown oil precipitated. About $2.5 \mathrm{ml}$ of benzene were distilled off in vacuo, then the mixture was cooled to $0^{\circ}$ and stirred for 10 $\mathrm{h}$ after addition of $10 \mathrm{ml}$ of $\mathrm{Et}_{2} \mathrm{O}$ : the amorphous redish precipitate was collected under $\mathrm{N}_{2}$ on a glas frit and washed with cold $\mathrm{Et}_{2} \mathrm{O}: 1.03 \mathrm{~g}(91 \%)$ pink crystals, melting range $60-70^{\circ} \mathrm{C}$.- IR (film): $\tilde{v}=2981(\mathrm{CH}) ; 1655$ $\mathrm{cm}^{-1}$ (CHO).- ${ }^{1} \mathrm{H}-\mathrm{NMR}: \delta$ (ppm) $=1.25\left(\mathrm{~d} ; \mathrm{J}=6.9 \mathrm{~Hz}, 3 \mathrm{H}, \mathrm{CHCH}_{3}\right), 2.13$ (s; $1.2 \mathrm{H}$, rotamere, $\left.\mathrm{NCH}_{3}\right), 2.20\left(\mathrm{~s} ; 1.8 \mathrm{H}\right.$, rotamere, $\left.\mathrm{NCH}_{3}\right), 4.32(\mathrm{~s} ; 2 \mathrm{H}$, $\mathrm{CH}_{2} \mathrm{Ph}$ ), 5.95 (q; $\mathrm{J}=6.9 \mathrm{~Hz}, 1 \mathrm{H}, \mathrm{CHCH}_{3}$ ), 6.90-7.22 (m; $5 \mathrm{H}$ aromat.), 7.52-8.34 (m; $6 \mathrm{H}$ aromat.), 9.56 (s; $1 \mathrm{H}, \mathrm{CHO})$.- MS (70 eV): m/z = 214 (14\%; educt, 38), $199\left(4, \mathrm{M}-\mathrm{CH}_{3}\right)^{+}, 185(12, \mathrm{M}-\mathrm{CHO})^{+}, 170 / 172$ (3, $\mathrm{C}_{6} \mathrm{H}_{5} \mathrm{CH}_{2} \mathrm{Br}$, thermal decomposition in the inlet system), 156 (12, M $\left.\mathrm{NCH}_{3} \mathrm{CHO}\right)^{+}, 91\left(100, \mathrm{C}_{7} \mathrm{H}_{7}\right)^{+}$.

\section{2-Benzyl-4-[1-(N-formyl-N-methylamino)-ethyl]-1,2,3,4- tetrahydroisoquinoline (44)}

To $0.40 \mathrm{~g}$ (1.04 mmole) 43 , dissolved in $20 \mathrm{ml}$ of absol. $\mathrm{MeOH}$, were added $0.50 \mathrm{~g} \mathrm{NaBH}_{4}$ at $0^{\circ}$. After addition of $1 \mathrm{ml}$ of water the reaction mixture was stirred for $15 \mathrm{~min}$ at $0^{\circ}$ and $16 \mathrm{~h}$ at room temp.- After acidification by $10 \mathrm{ml}$ of $2 \mathrm{~N} \mathrm{HCl}, \mathrm{MeOH}$ was evaporated in vacuo. After addition of $10 \mathrm{ml}$ of water and alkalization by dil. $\mathrm{NaOH}$ at $0^{\circ} 44$ was extracted by $\mathrm{Et}_{2} \mathrm{O}$. The org. phase was washed with brine and water, dried, and evaporated in vacuo at $40^{\circ}$ : yellow oil. Purification by cc $\left(\mathrm{CH}_{2} \mathrm{Cl}_{2}\right.$ :toluene:ethyl acetate $=8: 1: 2): 0.16 \mathrm{~g}(52 \%)$. IR (film): $\widetilde{v}=2927,2855$ $(\mathrm{CH}), 1669 \mathrm{~cm}^{-1}$ (CHO).- ${ }^{1} \mathrm{H}-\mathrm{NMR}: \delta(\mathrm{ppm})=1.27(\mathrm{~d} ; \mathrm{J}=6.0 \mathrm{~Hz}, 3 \mathrm{H}$, $\left.\mathrm{CH}-\mathrm{CH}_{3}\right), 2.24-2.57\left(\mathrm{~m} ; 2 \mathrm{H}, \mathrm{CH}_{2}\right), 2.78\left(\mathrm{~s} ; 3 \mathrm{H}, \mathrm{NCH}_{3}\right), 2.91-3.12(\mathrm{~m} ; 1 \mathrm{H}$, $\mathrm{CH}-\mathrm{C}$ - $-\mathrm{CH}_{3}$ ), 3.31 (AB-system, $\left.\mathrm{J}=5.4 \mathrm{~Hz}, 1 \mathrm{H}, \mathrm{CH}_{2}\right), 3.48(\mathrm{~s}, 2 \mathrm{H}$, $\mathrm{CH}_{2} \mathrm{Ph}$ ), 3.64 (AB-system, J = 5.4 Hz, $1 \mathrm{H}, \mathrm{CH}_{2}$ ), 3.96-4.21 (m, $1 \mathrm{H}, \mathrm{CH}_{2}-$ Cㄴ-CH), 6.77-7.48 (m, 9H aromat.), 8.00 (s, 1H, CHO).- MS (70 eV): m/z $=308\left(0.5 \%, \mathrm{M}^{+\cdot}\right), 305(1.5, \mathrm{M}-3 \mathrm{H})^{+}, 217\left(50, \mathrm{M}-\mathrm{C}_{7} \mathrm{H}_{7}\right)^{+}, 91(100$, $\left.\mathrm{C}_{7} \mathrm{H}_{7}\right)^{+}$.

\section{2-(3-Methoxyphenyl)-2-(2-methyldithiolan-2-yl)acetonitril (46) and 2-(3-Methoxyphenyl)-2-(2-methyldithian-2-yl)acetonitril (47)}

$1.0 \mathrm{~g}$ (5.0 mmole) nitril $\mathbf{4 5}^{16}$ ) were dissolved in $7 \mathrm{ml}$ of boiling $\mathrm{CH}_{2} \mathrm{Cl}_{2}$ by vigorous stirring. $1.50 \mathrm{ml}$ of ethane-1,2-dithiole or propane-1,3-dithiole for 47 , respectively, and then $1.00 \mathrm{ml}$ of $\mathrm{BF}_{3} \cdot \mathrm{Et}_{2} \mathrm{O}$ were added drop by drop at room temp. The reaction mixture was stirred for $24 \mathrm{~h}$ at $20^{\circ}$, mixed with $10 \mathrm{ml}$ of ice water, slightly alkalized by $5 \% \mathrm{NaOH}$ and extracted with $\mathrm{CH}_{2} \mathrm{Cl}_{2}(3 \times 5 \mathrm{ml})$. The org. phase was washed with brine, with water, dried, and evaporated: yellowish-greenish oil which was purified by twofold Kugelrohr distillation (150-180 0.02 torr).

46: $1.17 \mathrm{~g}(88 \%)$ colourless oil.- IR (film): $\widetilde{v}=2964(\mathrm{CH}), 2836(\mathrm{CH})$, $2242 \mathrm{~cm}^{-1}(\mathrm{CN})$.- ${ }^{1} \mathrm{H}-\mathrm{NMR}: \delta(\mathrm{ppm})=1.76\left(\mathrm{~s} ; 3 \mathrm{H}, \mathrm{CH}_{3}\right), 3.26-3.30(\mathrm{~m}$; $4 \mathrm{H}, \mathrm{SCH}_{2} \mathrm{CH}_{2} \mathrm{~S}$ ), 3.77 (s; $3 \mathrm{H}, \mathrm{OCH}_{3}$ ), 4.17 (s; $\left.1 \mathrm{H}, \mathrm{CH}-\mathrm{CN}\right), 6.75-7.45(\mathrm{~m}$, $4 \mathrm{H}$ aromat.).- MS $(70 \mathrm{eV}): \mathrm{m} / \mathrm{z}=224(0.3 \%, \mathrm{M}-\mathrm{HCN})^{+}, 146(1.5, \mathrm{M}-$ $\left.\mathrm{C}_{4} \mathrm{H}_{7} \mathrm{~S}_{2}\right)^{+}, 119\left(100, \mathrm{C}_{4} \mathrm{H}_{7} \mathrm{~S}_{2}\right)^{+}$.

47: $1.21 \mathrm{~g}(87 \%)$ colourless oil.- ${ }^{1} \mathrm{H}-\mathrm{NMR}: \delta(\mathrm{ppm})=1.59\left(\mathrm{~s} ; 3 \mathrm{H}, \mathrm{CH}_{3}\right)$, 1.74-2.21 (m; $\left.2 \mathrm{H}, \mathrm{SCH}_{2} \mathrm{C}_{2} \mathrm{CH}_{2} \mathrm{~S}\right), 2.72-3.03\left(\mathrm{~m} ; 4 \mathrm{H}, \mathrm{SC}_{2} \mathrm{CH}_{2} \mathrm{C}_{2} \mathrm{~S}\right)$, 3.79 (s; $3 \mathrm{H}, \mathrm{OCH}_{3}$ ), 4.44 (s; $\left.1 \mathrm{H}, \mathrm{CH}-\mathrm{CN}\right), 6.80-7.45$ (m; $4 \mathrm{H}$ aromat.).

\section{2-(3-Methoxyphenyl)-3-(1,4-dithiapentyl)-but-3-enyl-2-cyanide (48) and 2-(3-Methoxyphenyl)-3-(1,5-dithiahexyl)-but-3-enyl-2-cyanide (49)}

$30 \mathrm{ml}$ of THF and $\mathrm{Et}_{2} \mathrm{O}$ each and $3 \mathrm{ml}$ of freshly distilled TMEDA were cooled to $-70^{\circ}$. Then $10 \mathrm{ml}$ of $\mathrm{n}-\mathrm{BuLi}$ ( $15 \%$ in hexane) were slowly added under $\mathrm{N}_{2}$ followed by very slow addition of $2.00 \mathrm{~g}$ ( $\left.7.55 \mathrm{mmole}\right) 46$ (for 48) or $2.11 \mathrm{~g}$ ( $7.55 \mathrm{mmole}$ ) 47 (for 49) in $20 \mathrm{ml}$ of absol. THF. A tough red-brown precipitate was formed. The mixture was allowed to warm up to $-30^{\circ}$, then $3.38 \mathrm{~g} \mathrm{CH}_{3} \mathrm{I}$ in $20 \mathrm{ml}$ of $\mathrm{Et}_{2} \mathrm{O}$ were added in one portion, allowing temp. raise to $-10^{\circ}$. After $12 \mathrm{~h}$ stirring at room temp. the mixture was cooled to $-70^{\circ}, 10 \mathrm{ml}$ of satd. $\mathrm{NH}_{4} \mathrm{Cl}$ solution and $10 \mathrm{ml}$ of $\mathrm{EtOH}$ were added, and the mixture was stirred at room temp. until precipitated $\mathrm{NH}_{4} \mathrm{Cl}$ was dissolved. After addition of $5 \mathrm{ml}$ of brine the mixture was extracted 3 $\mathrm{x}$ with $5 \mathrm{ml} \mathrm{Et}_{2} \mathrm{O}$ each. Washing with water, drying, and evaporation afforded yellow oils which were purified by $\mathrm{cc}\left(\mathrm{CH}_{2} \mathrm{Cl}_{2}\right.$ :cyclohexane $=$ 2:1). 
48: $1.44 \mathrm{~g}(65 \%)$ colourless oil.- IR (film): $\widetilde{v}=2920(\mathrm{CH}), 2836(\mathrm{CH})$, $2240(\mathrm{CN}), 1601 \mathrm{~cm}^{-1}\left(\mathrm{H}_{2} \mathrm{C}=\mathrm{CH}\right) .{ }^{1} \mathrm{H}-\mathrm{NMR}: \delta(\mathrm{ppm})=1.94\left(\mathrm{~s} ; 3 \mathrm{H}, \mathrm{CH}_{3}\right)$, 2.10 (s; $3 \mathrm{H}, \mathrm{CH}_{3}$ ), 2.48-3.03 (m; $\left.4 \mathrm{H}, \mathrm{SCH}_{2} \mathrm{CH}_{2} \mathrm{~S}\right), 3.79$ (s; $3 \mathrm{H}, \mathrm{OCH}_{3}$ ), 5.20 (AB-system, J = 3.0 Hz, 1H, $\underline{\mathrm{HCH}}$ ), 5.77 (AB-system, $\mathrm{J}=3.0 \mathrm{~Hz}, 1 \mathrm{H}$, $\mathrm{HC} \underline{\mathrm{H}}), 6.72-7.37$ (m, 4H aromat.).- $\mathrm{MS}(70 \mathrm{eV}): \mathrm{m} / \mathrm{z}=293\left(2.5 \%, \mathrm{M}^{+^{*}}\right)$, $266(1, \mathrm{M}-\mathrm{HCN})^{+\bullet}, 246\left(1.5, \mathrm{M}-\mathrm{SCH}_{3}\right)^{+}, 218\left(3, \mathrm{M}-\mathrm{C}_{3} \mathrm{H}_{7} \mathrm{~S}\right)^{+}, 75(100$, $\left.\mathrm{C}_{3} \mathrm{H}_{7} \mathrm{~S}\right)^{+}$.

49: $1.58 \mathrm{~g} \mathrm{(68 \% )}$ colourless oil.- ${ }^{1} \mathrm{H}-\mathrm{NMR}: \delta(\mathrm{ppm})=1.95\left(\mathrm{~s} ; 3 \mathrm{H}, \mathrm{CH}_{3}\right)$, 2.07 (s; $3 \mathrm{H}, \mathrm{CH}_{3}$ ), 2.36-3.00 (m; $\left.2 \mathrm{H}, \mathrm{SCH}_{2} \underline{\mathrm{C}}_{2} \mathrm{CH}_{2} \mathrm{~S}\right), 2.53(\mathrm{t} ; \mathrm{J}=7.5 \mathrm{~Hz}$, $2 \mathrm{H}, \mathrm{CH}_{2} \mathrm{CH}_{2} \mathrm{CH}_{2}$ ), 2.82 (t; J = $7.5 \mathrm{~Hz}, 2 \mathrm{H}, \mathrm{SCH}_{2} \mathrm{CH}_{2} \mathrm{C}_{2} \mathrm{~S}$ ), 3.80 (s; $3 \mathrm{H}$, $\mathrm{OCH}_{3}$ ), 5.21 (AB-system, $\mathrm{J}=2.4 \mathrm{~Hz}, 1 \mathrm{H}, \underline{\mathrm{HCH}}$ ), 5.78 (AB-system, $\mathrm{J}=2.4$ $\mathrm{Hz}, 1 \mathrm{H}, \mathrm{HC} \underline{\mathrm{H}}), 6.82-7.51$ (m, $4 \mathrm{H}$ aromat.).

\section{2-(3-Methoxyphenyl)-2-(2-methyldithiolan-2-yl)-propionitril (50)}

To $0.10 \mathrm{~g}$ (0.34 mmole) 48 or $0.11 \mathrm{~g}(0.34$ mmole $) 49$, respectively, in $10 \mathrm{ml}$ of absol. $\mathrm{CH}_{2} \mathrm{Cl}_{2}$ were added under $\mathrm{N}_{2} 0.035 \mathrm{~g}$ ethane-1,2-dithiole and $0.1 \mathrm{~g} \mathrm{BF}_{3} \cdot 2 \mathrm{Et}_{2} \mathrm{O}$ at $0^{\circ}$. After stirring for $18 \mathrm{~h}$ at room temp. and addition of $5 \mathrm{ml}$ of ice water, the mixture was alkalized with $2 \mathrm{~N} \mathrm{NaOH}$ and extracted with $\mathrm{Et}_{2} \mathrm{O}$. The org. phase was washed $(2 \times 5 \mathrm{ml}$ of water), dried, and evaporated: brown oil which was purified by $\mathrm{cc}\left(\mathrm{CH}_{2} \mathrm{Cl}_{2}\right.$ :cyclohexane $=4: 1): 0.065 \mathrm{~g}(72 \%)$ colourless crystals, m.p. $60-62^{\circ}(\mathrm{MeOH})$.$\mathrm{C}_{14} \mathrm{H}_{17} \mathrm{NOS}_{2}$ (279.4) Calcd. C $60.2 \mathrm{H} 6.13 \mathrm{~N} 5.0$ Found C $60.4 \mathrm{H} 6.11 \mathrm{~N}$ 4.8.- IR (KBr): $\widetilde{v}=3005(\mathrm{CH}), 2936(\mathrm{CH}), 2236 \mathrm{~cm}^{-1}(\mathrm{CN}) .-{ }^{1} \mathrm{H}-\mathrm{NMR}: \delta$ $(\mathrm{ppm})=1.80\left(\mathrm{~s} ; 3 \mathrm{H}, \mathrm{CH}_{3}\right), 2.10\left(\mathrm{~s} ; 3 \mathrm{H}, \mathrm{CH}_{3}\right), 3.11-3.40(\mathrm{~m} ; 4 \mathrm{H}$, $\mathrm{SCH}_{2} \mathrm{CH}_{2} \mathrm{~S}$ ), 3.80 (s; $3 \mathrm{H}, \mathrm{OCH}_{3}$ ), 6.72-7.00 (m; $1 \mathrm{H}$ aromat.), 7.21-7.39 (m; $3 \mathrm{H}$ aromat.).- $\mathrm{MS}(70 \mathrm{eV}): \mathrm{m} / \mathrm{z}=279\left(0.1 \%, \mathrm{M}^{+*}\right), 161$ (1.3), 146 (1.0), $119\left(100, \mathrm{C}_{4} \mathrm{H}_{7} \mathrm{~S}_{2}\right)^{+}$.

\section{2-(3-Methoxyphenyl)-2-(2-methyldithiolan-2-yl)-propylamine (51)}

$1.00 \mathrm{~g}$ (3.58 mmole) $\mathbf{5 0}$ in $25 \mathrm{ml}$ of absol. THF:1,2-dimethoxyethane = $1: 1$ were cooled to $0^{\circ}$. Then $7.2 \mathrm{ml}$ THF- $\mathrm{BH}_{3}$ complex ( 1 molar in THF) were added carefully by a syringe and the mixture was stirred at $0^{\circ}$ for 15 $\min$ at room temp., $1 \mathrm{~h}$ at $65^{\circ}$, and $10 \mathrm{~h}$ at $105^{\circ}$ (tlc control).- For work-up the mixture was cooled to $0^{\circ}, 5 \mathrm{ml}$ of cold EtOH were added slowly, $\mathrm{NH}_{3}$ rendered the mixture sligthly alkaline, and $\mathbf{5 1}$ was extracted with $\mathrm{Et}_{2} \mathrm{O}$. Drying and evaporation yielded a brownish oil; purification by cc (ethyl acetate: $\mathrm{EtOH}=4: 1): 0.133 \mathrm{~g}$ brown oil.- IR (film): $\tilde{\mathrm{v}}=3367(\mathrm{NH}), 2920$ $\mathrm{cm}^{-1}(\mathrm{CH}) .{ }^{1} \mathrm{H}-\mathrm{NMR}: \delta(\mathrm{ppm})=1.67\left(\mathrm{~m} ; 8 \mathrm{H}, 2 \times \mathrm{CH}_{3}\right.$ and $\left.\mathrm{C}_{2} \mathrm{NH}_{2}\right)$, 2.87-3.23 (m; $\left.6 \mathrm{H}, \mathrm{SCH}_{2} \mathrm{CH}_{2} \mathrm{~S}, \mathrm{CH}_{2} \mathrm{NH}_{2}\right), 3.73\left(\mathrm{~s} ; 3 \mathrm{H}, \mathrm{OCH}_{3}\right), 6.65-7.37$ (m; $4 \mathrm{H}$ aromat.).- $\mathrm{MS}(70 \mathrm{eV}): \mathrm{m} / \mathrm{z}=283\left(2.5 \%, \mathrm{M}^{+\cdot}\right), 254(10, \mathrm{M}$ $\left.\mathrm{CH}_{2} \mathrm{NH}\right)^{+}, 293\left(10, \mathrm{M}-\mathrm{C}_{4} \mathrm{H}_{7} \mathrm{~S}_{2}\right)^{+}, 119\left(100, \mathrm{C}_{4} \mathrm{H}_{7} \mathrm{~S}_{2}\right)^{+}$.

\section{$N$-Acetyl-2-(3-methoxyphenyl)-2-(2-methyldithiolan-2-yl)propylamine} (52)

$0.20 \mathrm{~g}$ (0.71 mmole) 51 in $5 \mathrm{ml}$ of absol. $\mathrm{CH}_{2} \mathrm{Cl}_{2}$ were mixed with $0.55 \mathrm{~g}$ acetyl chloride at $0^{\circ}$ under $\mathrm{N}_{2}$ and stirred at $0^{\circ}$ and at room temp. for $1 \mathrm{~h}$ each. The red mixture was cooled to $0^{\circ}$, neutralized by $\mathrm{N} \mathrm{NaOH}$ and extracted with $\mathrm{Et}_{2} \mathrm{O}(3 \times 5 \mathrm{ml})$. The org. phase was washed with satd. $\mathrm{NaHCO}_{3}$ solution, with water, dried, and evaporated: yellow oil, purification by cc (ethyl acetate: $\mathrm{MeOH}=3: 2$ ): $0.19 \mathrm{~g}(82 \%)$. - IR (film): $\widetilde{v}=3314$ (NH), $3081(\mathrm{NH}), 2973(\mathrm{CH}), 2925(\mathrm{CH}), 1655 \mathrm{~cm}^{-1}(\mathrm{CO}) .{ }^{1} \mathrm{H}-\mathrm{NMR}: \delta$ $(\mathrm{ppm})=1.63\left(\mathrm{~s} ; 3 \mathrm{H}, \mathrm{CH}_{3}\right), 1.70\left(\mathrm{~s} ; 3 \mathrm{H}, \mathrm{CH}_{3}\right), 1.83\left(\mathrm{~s} ; 3 \mathrm{H}, \mathrm{CH}_{3}\right), 2.97-3.40$ (m; $4 \mathrm{H}, \mathrm{SCH}_{2} \mathrm{CH}_{2} \mathrm{~S}$ ), 3.82 (s; $3 \mathrm{H}, \mathrm{OCH}_{3}$ ), 4.20 (d; J = 6 Hz, $2 \mathrm{H}, \mathrm{C}_{2} \mathrm{NH}$ ), $5.60\left(\mathrm{t} ; \mathrm{J}=6 \mathrm{~Hz}, 1 \mathrm{H}, \mathrm{CH}_{2} \mathrm{NH}\right), 6.75-7.40$ (m; $4 \mathrm{H}$ aromat.).- $\mathrm{MS}(70 \mathrm{eV})$ : $\mathrm{m} / \mathrm{z}=325\left(0.5 \%, \mathrm{M}^{+\bullet}\right), 254\left(1, \mathrm{M}-\mathrm{CH}_{2} \mathrm{NHCOCH}_{3}\right)^{+}, 119\left(100, \mathrm{C}_{4} \mathrm{H}_{7} \mathrm{~S}_{2}\right)^{+}$.

6-Methoxy-1,4-dimethyl-4-(2-methyldithiolan-2-yl)-3,4-

dihydroisoquinoline (53)

In a throrougly dried flask $0.44 \mathrm{~g}$ (1.34 mmole) 52 in $10 \mathrm{ml}$ of absol. acetonitril were cooled to $0^{\circ}$ under $\mathrm{N}_{2}$ and slowly mixed with $1.5 \mathrm{ml}$ of freshly distilled $\mathrm{POCl}_{3}$ in $2 \mathrm{ml}$ of absol. acetonitril, followed by stirring for
$24 \mathrm{~h}$ at room temp. Then about $6 \mathrm{ml}$ of acetonitril were distilled off at the oil pump. After addition of $6 \mathrm{ml}$ of acetone, the dark brown solution was neutralized by addition of $\mathrm{NaOH}$, then alkalized by $\mathrm{NaHCO}_{3}$-solution. The separated crystals were washed with cold $\mathrm{CH}_{2} \mathrm{Cl}_{2}$ and discarded (inorganic material). The filtrate was extracted with $\mathrm{Et}_{2} \mathrm{O}(3 \times 5 \mathrm{ml})$, the ether layer was combined with the $\mathrm{CH}_{2} \mathrm{Cl}_{2}$-washing, dried and evaporated: dark brown oil which was directly reduced to the tetrahydroisoquinoline 54 .

53: $0.35 \mathrm{~g}(85 \%)$.- UV: $\lambda \max (\log \varepsilon)=202(4.23), 248(3.50), 276 \mathrm{~nm}$ (3.68).- IR (film): $\widetilde{v}=2923,2836(\mathrm{CH}), 1640 \mathrm{~cm}^{-1} .{ }^{1} \mathrm{H}-\mathrm{NMR}: \delta(\mathrm{ppm})=$ $1.58\left(\mathrm{~s} ; 3 \mathrm{H}, \mathrm{CH}_{3}\right), 1.67\left(\mathrm{~s} ; 3 \mathrm{H}, \mathrm{CH}_{3}\right), 2.26\left(\mathrm{~s} ; 3 \mathrm{H}, \mathrm{CH}_{3}\right), 2.95-3.38(\mathrm{~m} ; 4 \mathrm{H}$, $\mathrm{SCH}_{2} \mathrm{CH}_{2} \mathrm{~S}$ ), 3.37 (AB-system, J = $15 \mathrm{~Hz}, 1 \mathrm{H}, \underline{\mathrm{HCH}}$ ), 3.80 (s; $3 \mathrm{H}, \mathrm{OCH}_{3}$ ), 4.26 (AB-system, J = $15 \mathrm{~Hz}, 1 \mathrm{H}, \mathrm{HC} \underline{\mathrm{H}}$ ), 6.78 (dd; $\mathrm{J}_{1}=9 \mathrm{~Hz}, \mathrm{~J}_{2}=2.4 \mathrm{~Hz}$, 1H, 7-H), 7.20 (d; J = 2.4 Hz, 1H, 5-H), 7.41 (d; J = 9 Hz, 1H, 8-H).- MS $(70 \mathrm{eV}): \mathrm{m} / \mathrm{z}=307\left(0.2 \%, \mathrm{M}^{+*}\right), 305(1.5, \mathrm{M}-2 \mathrm{H})^{+*}, 189(40, \mathrm{M}+\mathrm{H}-$ $\left.\mathrm{C}_{4} \mathrm{H}_{7} \mathrm{~S}_{2}\right)^{+\bullet}, 119\left(100, \mathrm{C}_{4} \mathrm{H}_{7} \mathrm{~S}_{2}\right)^{+}$.

\section{6-Methoxy-1,4-dimethyl-4-(2-methyldithiolan-2-yl)-1,2,3,4-} tetrahydroisoquinoline (54)

$0.34 \mathrm{~g}$ (1.09 mmole) crude dihydroisoquinoline $53 \mathrm{in} 10 \mathrm{ml}$ of absol. $\mathrm{MeOH}$ were cooled to $0^{\circ}$, mixed with $0.30 \mathrm{~g} \mathrm{NaBH}_{4}$ in portions, and stirred for $4 \mathrm{~h}$ at $0^{\circ}$. The excess of $\mathrm{NaBH}_{4}$ was destroyed by about $5 \mathrm{ml}$ of $2 \mathrm{~N}$ $\mathrm{HCl}, \mathrm{MeOH}$ was distilled off at $20^{\circ}$ and the aqueous phase was neutralized by $\mathrm{NaHCO}_{3}$. Extraction with $\mathrm{Et}_{2} \mathrm{O}$ and cc (ethyl acetate:EtOH = 3:2) afforded $0.30 \mathrm{~g}(88 \%)$ of a colourless oil.- IR (film): $\widetilde{v}=3330(\mathrm{NH}), 2967$, 2925, $2834 \mathrm{~cm}^{-1}(\mathrm{CH}) .{ }^{1} \mathrm{H}-\mathrm{NMR}: \delta(\mathrm{ppm})=1.45(\mathrm{~d} ; \mathrm{J}=6.9 \mathrm{~Hz}, 3 \mathrm{H}$, $\left.\mathrm{CHCH}_{3}\right), 1.48\left(\mathrm{~s} ; 3 \mathrm{H}, \mathrm{CH}_{3}\right), 1.86\left(\mathrm{~s} ; 3 \mathrm{H}, \mathrm{CH}_{3}\right), 2.13(\mathrm{~s} ; 1 \mathrm{H}, \mathrm{NH}$, exchangeable), 2.87 (AB-system, J = 11.7 Hz, 1H, $\underline{\mathrm{HCH}}$ ), 3.04-3.30 (m; $4 \mathrm{H}$, $\mathrm{SCH}_{2} \mathrm{CH}_{2} \mathrm{~S}$ ), 3.47 (AB-system, $\mathrm{J}=11.7 \mathrm{~Hz}, 1 \mathrm{H}, \mathrm{HC} \underline{\mathrm{H}}$ ), 3.79 (s; $3 \mathrm{H}$, $\mathrm{OCH}_{3}$ ), 4.07 (q; J $=6.9 \mathrm{~Hz}, 1 \mathrm{H}, \mathrm{CHCH}_{3}$ ), 6.67-7.44 (m; 3H aromat.).- $\mathrm{MS}$ $(70 \mathrm{eV}): \mathrm{m} / \mathrm{z}=309\left(1.5 \%, \mathrm{M}^{+\cdot}\right), 294\left(1.4, \mathrm{M}-\mathrm{CH}_{3}\right)^{+}, 191(40, \mathrm{M}+\mathrm{H}-$ $\left.\mathrm{C}_{4} \mathrm{H}_{7} \mathrm{~S}_{2}\right)^{+\bullet}, 119\left(100, \mathrm{C}_{4} \mathrm{H}_{7} \mathrm{~S}_{2}\right)^{+}$.

\section{4-Acetyl-6-methoxy-1,4-dimethyl-1,2,3,4-tetrahydroisoquinoline (55)}

To $0.34 \mathrm{~g}$ (1.1 mmole) $54 \mathrm{in} 10 \mathrm{ml}$ of freshly distilled $\mathrm{CHCl}_{3}$ and $12 \mathrm{ml}$ of $\mathrm{MeOH}$ were added $0.49 \mathrm{~g} \mathrm{Hg}\left(\mathrm{ClO}_{4}\right)_{2} \cdot 2 \mathrm{H}_{2} \mathrm{O}$ in $8 \mathrm{ml}$ of $\mathrm{MeOH}$ drop by drop, affording a white precipitate. The suspension was stirred under $\mathrm{N}_{2}$ for $1 \mathrm{~h}$ at room temp. and for $48 \mathrm{~h}$ at $40^{\circ}$. After filtration and washing of the solid with $\mathrm{CHCl}_{3}$ and $\mathrm{MeOH}$, the solvents were evaporated at $20^{\circ}$. The remaining brown oil was dissolved in $2 \mathrm{~N} \mathrm{HCl}$ of $0^{\circ}$, stirred for $1 \mathrm{~h}$, neutralized by $\mathrm{NaHCO}_{3}$, and the base was extracted by $\mathrm{Et}_{2} \mathrm{O}$. Usual purification $\left(\mathrm{CH}_{2} \mathrm{Cl}_{2}\right.$ :acetone: $\left.\mathrm{MeOH}=6: 3: 1\right)$ afforded $0.12 \mathrm{~g} \mathrm{(47 \% )}$ colourless oil.- IR (film): $\widetilde{v}=3336$ (NH), 2965, 2929, 2834 (CH), $1702 \mathrm{~cm}^{-1}$ (CO).${ }^{1} \mathrm{H}-\mathrm{NMR}: \delta(\mathrm{ppm})=1.36\left(\mathrm{~s} ; 3 \mathrm{H}, \mathrm{CH}_{3}\right), 1.40\left(\mathrm{~d} ; \mathrm{J}=6.3 \mathrm{~Hz}, 3 \mathrm{H}, \mathrm{CHC}_{3}\right)$, $2.08\left(\mathrm{~s} ; 3 \mathrm{H}, \mathrm{CH}_{3}\right), 2.26(\mathrm{~s} ; 1 \mathrm{H}, \mathrm{NH}$, exchangeable), 2.83 (AB-system, $\mathrm{J}=$ $11.7 \mathrm{~Hz}, 1 \mathrm{H}, \underline{\mathrm{HCH}}$ ), 3.54 (AB-system, J = $11.7 \mathrm{~Hz}, 1 \mathrm{H}, \mathrm{HC} \underline{\mathrm{H}}$ ), 3.75 (s; $3 \mathrm{H}, \mathrm{OCH}_{3}$ ), 4.07 (q; $\mathrm{J}=6.3 \mathrm{~Hz}, 1 \mathrm{H}, \mathrm{CHCH}_{3}$ ), 6.65-7.21 (m; $3 \mathrm{H}$ aromat.).MS $(70 \mathrm{eV}): \mathrm{m} / \mathrm{z}=233\left(33 \%, \mathrm{M}^{+*}\right), 218\left(100, \mathrm{M}-\mathrm{CH}_{3}\right)^{+}, 190(30, \mathrm{M}-$ $\left.\mathrm{COCH}_{3}\right)^{+}, 189\left(50, \mathrm{M}-\mathrm{COCH}_{3}-\mathrm{H}\right)^{+\cdot}, 43\left(20, \mathrm{COCH}_{3}\right)^{+}$.

\section{4-(1-Methoxyiminoethyl)-6-methoxy-1,4-dimethyl-1,2,3,4- tetrahydroisoquinoline (56)}

The mixture of $0.10 \mathrm{~g}$ ( $0.83 \mathrm{mmole}) 55$ and $0.12 \mathrm{~g}$ ( $2.4 \mathrm{mmole}) O$ methyl-hydroxylammonium chloride in $10 \mathrm{ml}$ of pyridine was heated to reflux under $\mathrm{N}_{2}$ for $2 \mathrm{~h}$. Pyridine was removed at the oil pump at $60^{\circ}$, the residue was dissolved in $3 \mathrm{ml}$ of $2 \mathrm{~N} \mathrm{HCl}$ and extracted with $\mathrm{Et}_{2} \mathrm{O}$. The aqueous phase was alkalized by dil. $\mathrm{NaOH}$ and extracted with ether. Purification by $\mathrm{cc}\left(\mathrm{CH}_{2} \mathrm{Cl}_{2}\right.$ :acetone: $\left.\mathrm{MeOH}=6: 3: 1\right): 0.082 \mathrm{~g}(76 \%)$ colourless oil.- IR (film): $\widetilde{v}=3336$ (NH), $2932(\mathrm{CH}), 1611 \mathrm{~cm}^{-1}(\mathrm{C}=\mathrm{N})$ ). ${ }^{1} \mathrm{H}-\mathrm{NMR}: \delta$ $(\mathrm{ppm})=1.39\left(\mathrm{~s} ; 3 \mathrm{H}, \mathrm{CH}_{3}\right), 1.43\left(\mathrm{~d} ; \mathrm{J}=6.3 \mathrm{~Hz}, 3 \mathrm{H}, \mathrm{CHCH}_{3}\right), 1.73(\mathrm{~s} ; 3 \mathrm{H}$, $\mathrm{CH}_{3}$ ), 1.94 (s; 1H, NH), 2.79 (AB-system, J = 11.7 Hz, 1H, $\underline{\mathrm{HCH}}$ ), 3.40 (AB-system, J = $11.7 \mathrm{~Hz}, 1 \mathrm{H}, \mathrm{HCH}), 3.76$ (s; $3 \mathrm{H}, \mathrm{NOCH}_{3}$ ), 3.90 (s; $3 \mathrm{H}$, 
$\left.\mathrm{OCH}_{3}\right), 4.12$ (q; J = 6.3 Hz, $1 \mathrm{H}, \mathrm{CHCH}_{3}$ ), 6.65-7.50 (m; 3H aromat.).- $\mathrm{MS}$ $(70 \mathrm{eV}): \mathrm{m} / \mathrm{z}=262\left(0.6 \%, \mathrm{M}^{+\bullet}\right), 259(10, \mathrm{M}-3 \mathrm{H})^{+}, 247\left(60, \mathrm{M}-\mathrm{CH}_{3}\right)^{+}$, $231\left(75, \mathrm{M}-\mathrm{OCH}_{3}\right)^{+}, 190\left(100, \mathrm{M}-\mathrm{H}_{3} \mathrm{CCNOCH}_{3}\right)^{+}$.

There is no indication for diastereomers.

\section{2-(3-Methoxyphenyl)-3-(2,5-dithiapentyl)-crotononitril (57)}

$750 \mathrm{ml}$ of absol. THF and $750 \mathrm{ml}$ of absol. $\mathrm{Et}_{2} \mathrm{O}$ were cooled to $-70^{\circ}$ in a three necked flask and mixed with $250 \mathrm{ml}$ cooled n-BuLi (15\% in hexane) under $\mathrm{N}_{2}$. With vigorous stirring $50.0 \mathrm{~g}(0.19 \mathrm{~mole})$ nitril 46 in $100 \mathrm{ml}$ of absol. THF were added drop by drop keeping the temp. at $-70^{\circ}$. Then the cooling device was removed and the mixture was allowed to reach $-30^{\circ}$. At this temp. $84.5 \mathrm{~g} \mathrm{CH}_{3} \mathrm{I}(0.60 \mathrm{~mole})$ in $200 \mathrm{ml}$ of absol. $\mathrm{Et}_{2} \mathrm{O}$ were added in one portion and the mixture was stirred at room temp. for $2 \mathrm{~h}$. After cooling to $-70^{\circ} 250 \mathrm{ml}$ of cooled $\mathrm{EtOH}$ and $250 \mathrm{ml}$ of satd. $\mathrm{NH}_{4} \mathrm{Cl}$ solution were added.- After reaching $0^{\circ}$ water was added slowly until precipitated $\mathrm{NH}_{4} \mathrm{Cl}$ had dissolved. After mixing with $100 \mathrm{ml}$ of brine, extraction with $\mathrm{Et}_{2} \mathrm{O}$, and purification of crude brown 57 by cc $\left(\mathrm{CH}_{2} \mathrm{Cl}_{2}\right.$ :cyclohexane = 2:1), $17.4 \mathrm{~g} \mathrm{(33 \% )}$ colourless oil were obtained. IR (film): $\tilde{\mathrm{v}}=2919(\mathrm{CH})$; $2836(\mathrm{CH}) ; 2203(\mathrm{CN}) ; 1599 \mathrm{~cm}^{-1}(\mathrm{C}=\mathrm{C})$.- ${ }^{1} \mathrm{H}-\mathrm{NMR}$ (E/Z-mixture): $\delta$ $(\mathrm{ppm})=2.05\left(\mathrm{~s} ; 2 \mathrm{H}, \mathrm{CH}_{3}\right), 2.15\left(\mathrm{~s} ; 1 \mathrm{H}, \mathrm{CH}_{3}\right), 2.20\left(\mathrm{~s} ; 1 \mathrm{H}, \mathrm{CH}_{3}\right), 2.51(\mathrm{~s} ;$ $\left.2 \mathrm{H}, \mathrm{CH}_{3}\right), 2.52-3.32\left(\mathrm{~m} ; 4 \mathrm{H}, \mathrm{SCH}_{2} \mathrm{CH}_{2} \mathrm{~S}\right), 3.74\left(\mathrm{~s} ; 3 \mathrm{H}, \mathrm{OCH}_{3}\right), 6.73-7.52$ (m; $4 \mathrm{H}$ aromat.).- $\mathrm{MS}(70 \mathrm{eV}): \mathrm{m} / \mathrm{z}=279\left(16 \%, \mathrm{M}^{+*}\right), 219(18, \mathrm{M}-$ $\left.\mathrm{C}_{2} \mathrm{H}_{4} \mathrm{~S}\right)^{+}, 204\left(19, \mathrm{M}-\mathrm{C}_{2} \mathrm{H}_{4} \mathrm{SCH}_{3}\right)^{+}, 75\left(100, \mathrm{C}_{2} \mathrm{H}_{4} \mathrm{SCH}_{3}\right)^{+}$.

Preparation of 48 from 57 follows the preparation of 48 from 46: 57: $2.00 \mathrm{~g}$ (7.2 mmole).- $48: 1.60 \mathrm{~g}, 72 \%$.

\section{2-(3-Methoxyphenyl)-2-thioacetyl-propionitril (58)}

To the solution of $0.850 \mathrm{~g}$ ( 3.20 mmole $) 46 \mathrm{in} 10 \mathrm{ml}$ of absol. THF: $\mathrm{Et}_{2} \mathrm{O}$ $=1: 1$ were added under $\mathrm{N}_{2} 1.95 \mathrm{ml}$ of $\mathrm{n}-\mathrm{BuLi}$ ( $15 \%$ in hexane) and $5 \mathrm{ml}$ of $\mathrm{Et}_{2} \mathrm{O}$ at $-70^{\circ}$. The cooling bath was removed, the mixture was stirred for 15 $\mathrm{min}$ at room temp., $0.454 \mathrm{~g} \mathrm{CH}_{3} \mathrm{I}$ in $5 \mathrm{ml}^{\text {of }} \mathrm{Et}_{2} \mathrm{O}$ were added drop by drop, and the mixture was stirred at room temp. for $10 \mathrm{~h}$. After addition of $10 \mathrm{ml}$ of satd. $\mathrm{NH}_{4} \mathrm{Cl}$ solution at $0^{\circ}$ the mixture was stirred for $15 \mathrm{~min}$ and extracted with $\mathrm{Et}_{2} \mathrm{O}$. Usual work-up yielded a yellow oil which was purified by cc $\left(\mathrm{CH}_{2} \mathrm{Cl}_{2}\right.$ :cyclohexane $\left.=2: 1\right): 0.37 \mathrm{~g}(53 \%)$ colourless oil.- $\mathrm{IR}$ (film): $\widetilde{\mathbf{v}}=3002(\mathrm{CH}) ; 2202(\mathrm{CN}) ; 1600 \mathrm{~cm}^{-1}(\mathrm{CS}) .{ }^{1} \mathrm{H}-\mathrm{NMR}: \delta(\mathrm{ppm})=$ $2.31\left(\mathrm{~s} ; 3 \mathrm{H}, \mathrm{CH}_{3}\right) ; 2.50\left(\mathrm{~s} ; 3 \mathrm{H}, \mathrm{CH}_{3}\right) ; 3.74\left(\mathrm{~s} ; 3 \mathrm{H}, \mathrm{OCH}_{3}\right) ; 6.70-7.47(\mathrm{~m}$; $4 \mathrm{H}$ aromat.).- MS $(70 \mathrm{eV}): \mathrm{m} / \mathrm{z}=219\left(50 \%, \mathrm{M}^{+\bullet}\right) ; 204\left(100, \mathrm{M}-\mathrm{CH}_{3}\right)^{+}$; $189\left(50,204-\mathrm{CH}_{3}\right)^{+\bullet} ; 171(30)$.

B. Dehydrogenation experiments

\section{Standard conditions}

The tetraline quantity necessary for the respective compound was freed from $\mathrm{O}_{2}$ by bubling $\mathrm{N}_{2}$ into it at $100^{\circ}$ for $15 \mathrm{~min}$.- After cooling to room temp. the compound to be tested and $\mathrm{Pd} / \mathrm{C}(10 \%)$ were added. After heating for 1-2 $\mathrm{h}$ under reflux tetraline was distilled off in a Kugelrohr apparatus. The pertinent product was purified by cc.

\section{4-(N-Formyl-pyrrolidin-2-yl)-isoquinoline (9a)}

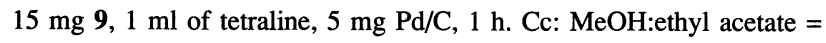
8:2.- Data see above.

\section{4-(N-Formyl-piperidin-2-yl)-isoquinoline (10a)}

$15 \mathrm{mg} \mathrm{10}, 1 \mathrm{ml}$ of tetraline, $5 \mathrm{mg} \mathrm{Pd} / \mathrm{C}, 1 \mathrm{~h}$. (Cc: cf. 9a).- Data see above.

\section{6,7-Dimethoxy-1,4-dimethylisoquinoline (12)}

run A: $5.0 \mathrm{mg} \mathrm{11,} 1 \mathrm{ml}$ of tetraline, $5.0 \mathrm{mg} \mathrm{Pd} / \mathrm{C}, 1 \mathrm{~h} .-\mathrm{Cc}$ : $\mathrm{CHCl}_{3}$ :EtOH:conc. $\mathrm{NH}_{3}=85: 14: 1 .-4.1 \mathrm{mg}$ brownish oil.- $\mathrm{MS}(70 \mathrm{eV})$ : $\mathrm{m} / \mathrm{z}=217\left(100 \%, \mathrm{M}^{+*}\right), 202\left(16, \mathrm{M}-\mathrm{CH}_{3}\right)^{+}$.- UV (qual.): $\max =201 ; 238$; $280 ; 326 \mathrm{~nm}$.

run B: same conditions as for run $\mathrm{A}$, but no $\mathrm{Pd} / \mathrm{C}$ : $4.0 \mathrm{mg} 12$.
6,7-Dimethoxy-1-methyl-3,4-dihydroisoquinoline (14): Lit. ${ }^{2)}$.

6,7-Dimethoxy-1-(3,4-methylendioxybenzyl)-4-(N-methylpyrrol-

2-yl)-isoquinoline (16a)

$200 \mathrm{mg} 15$ or $200 \mathrm{mg} \mathrm{16}$, respectively, $6 \mathrm{ml}$ of tetraline, $50 \mathrm{mg} \mathrm{Pd} / \mathrm{C}, 2$ h.- Solvents were evaporated: brownish oil. Cc: ethyl acetate, distillation (Kugelrohr, $0.05 \mathrm{~mm} \mathrm{Hg}, 250^{\circ}$ ): $150 \mathrm{mg}$ yellow crystals (72\%).- m.p. 69$71^{\circ}$.- 16a-picrate: m.p. $172-173^{\circ}$ (dec.).- 16a-picrate: $\mathrm{C}_{30} \mathrm{H}_{25} \mathrm{~N}_{5} \mathrm{O}_{11}$ (631.5) Calcd. C 57.0 H 3.99 N 11.1 Found C 56.9 H 4.09 N 10.9.- UV: $\lambda \max$ $(\log \varepsilon)=216(4.49), 287 \mathrm{~nm}(4.08)$.- IR $(\mathrm{KBr}): \widetilde{v}=2795 \mathrm{~cm}^{-1}\left(\mathrm{NCH}_{3}\right)$.MS (70 eV): m/z = $402\left(79 \%, \mathrm{M}^{+\bullet}\right), 401$ (100), 386 (7), 385 (14), 298 (14), 282 (23), 268 (94), 186 (20).- ${ }^{1} \mathrm{H}-\mathrm{NMR}: \delta(\mathrm{ppm})=3.38(\mathrm{~s} ; 3 \mathrm{H}$, $\left.\mathrm{NCH}_{3}\right) ; 3.87\left(\mathrm{~s} ; 3 \mathrm{H}, \mathrm{OCH}_{3}\right) ; 3.95\left(\mathrm{~s} ; 3 \mathrm{H}, \mathrm{OCH}_{3}\right) ; 4.36\left(\mathrm{~s} ; 2 \mathrm{H}, \mathrm{OCH}_{2} \mathrm{O}\right)$; 5.87 (s; $2 \mathrm{H}, \mathrm{PhCH}_{2}$ ), 6.45-7.06 (m; $8 \mathrm{H}$ aromat.), 8.1 (s; $\left.1 \mathrm{H}, 3-\mathrm{H}\right)$.

\section{4-Ethylisoquinoline $(\mathbf{1 8})^{5)}$}

$36 \mathrm{mg} 17$ or $45 \mathrm{mg} \mathrm{19}$, respectively, $1 \mathrm{ml}$ of tetraline, $30 \mathrm{mg} \mathrm{Pd} / \mathrm{C}$ or 45 $\mathrm{mg}$, respectively, 1 h.- Cc: ethyl acetate.- $\mathrm{C}_{11} \mathrm{H}_{11} \mathrm{~N}$ (157.2).- IR (film): $\widetilde{v}=$ $2950 \mathrm{~cm}^{-1}(\mathrm{CH}) .{ }^{1} \mathrm{H}-\mathrm{NMR}(250 \mathrm{MHz}): \delta(\mathrm{ppm})=1.39(\mathrm{t} ; \mathrm{J}=7.54 \mathrm{~Hz}, 3 \mathrm{H}$, $\mathrm{CH}_{2}-\underline{\mathrm{CH}}_{3}$ ), 3.07 (q; $\mathrm{J}=7.54 \mathrm{~Hz}, 2 \mathrm{H}, \mathrm{C}_{2}-\mathrm{CH}_{3}$ ), 7.57-8.03 (m; $4 \mathrm{H}$ aromat.), 8.40 (s; $1 \mathrm{H}$ aromat.), 9.13 (s; $1 \mathrm{H}$ aromat.).- $\mathrm{MS}: \mathrm{m} / \mathrm{z}=157$ (73\%, $\left.\mathrm{M}^{+\bullet}\right), 142\left(100, \mathrm{M}-\mathrm{CH}_{3}\right)^{+}$.- UV: $\lambda \max (\log \varepsilon)=217$ (4.33); 271 (3.27); 308 (3.15); $322 \mathrm{~nm}(3.25)$.

\section{4-(1-Aminoethyl)-6-methoxy-1,4-dimethyl-3,4-dihydroisoquinoline (21)}

$31 \mathrm{mg} \mathrm{20}, 1 \mathrm{ml}$ of tetraline, $13 \mathrm{mg} \mathrm{Pd} / \mathrm{C}, 1$ h.- UV (MeOH, 0.1HCl): $\lambda$ $\max (\log \varepsilon)=202$ (4.15); 258 (3.70); $315 \mathrm{~nm}(3.75)$.- $\mathrm{MS}(70 \mathrm{eV}): \mathrm{m} / \mathrm{z}=$ $232\left(1.1 \% ; \mathrm{M}^{+\bullet}\right), 188\left(3 ; \mathrm{M}-\mathrm{H}_{3} \mathrm{CCHNH}_{2}\right)^{+}, 187\left(8 ; \mathrm{M}-\mathrm{H}_{3} \mathrm{CCHNH}_{2}-\right.$ $\mathrm{H})^{+\bullet}, 44\left(24 ; \mathrm{H}_{3} \mathrm{CCHNH}_{2}\right)^{+}$.

\section{References}

1 W. Wiegrebe, S. Mahboobi, G. Dannhardt, K.K. Mayer, E. Eibler, Chimia 1981, 35, 288-289.

2 S. Mahboobi, E. Seidl, E. Eibler, W. Wiegrebe, Arch. Pharm. (Weinheim) 1992, 325, 679-683.

3 W. Wykypiel, D. Seebach, Tetrahedron Lett. 1980, 21, 1927-1930.

4 S. Mahboobi, E.Ch. Fischer, E. Eibler, W. Wiegrebe, Arch. Pharm. (Weinheim) 1988, 321, 423-424.

5 G. Jones, J. Chem. Soc. 1960, 1918-1923.

6 J.J. Padbury, H.G. Lindwall, J. Am. Chem. Soc. 1945, 67, 1268-1270.

7 A. Giovanni, D. Savoia, A. Umani-Ronchi, J. Org. Chem. 1989, 54, 228-234.

8 R. Henning, F. Lehr, D. Seebach, Helv. Chim. Acta, 1976, 59, 22132217.

9 M.M. Baradarani, L. Dalton, F. Heatley, D. Cohylakis, J.A. Joule, J. Chem. Soc. Perkin Trans. 1 1985, 1503-1508.

10 W.H. Hartung, R. Simonoff, Org. React. 7 1953, 275-278.

11 R. Grewe, A. Mondon, E. Nolte, Liebigs Ann. Chem. 1949, 564, 161198.

12 M.P. Cava, D.R. Dalton, J. Org. Chem. 1966 31, 1281-1283.

13 H. Yagi, D.R. Thakker, R.E. Lehr, D.M. Jerina, J. Org. Chem. 1979, 44, 3442-3444.

14 M. Amat, A. Linares, J. Bosch, J. Org. Chem. 1990, 55, 6299-6312.

15 M. Amat, M. Alvarez, J. Bonjoch, N. Casamitjana, J. Gracia, R. Lavilla, X. Garcias, J. Bosch, Tetrahedron Lett. 1990, 31, 3453-3456.

16 T. Koyama, T. Hirota, M. Toda, K. Iwai, M. Minami, M. Yamato, Yakugaku Zasshi 1969, 89, 1334-1337; Chem. Abstr. 1970, 72, 43603c.

17 E. Fujita, Y. Nagao, K. Kaneko, Chem. Pharm. Bull. 1978, 26, 37433751 .

18 W. Wagner, Diplomarbeit, Univ. Regensburg, 1991, p. 37-38. 